\title{
The Evolution of Binary Systems
}

\author{
Philipp Podsiadlowski ${ }^{1}$
}

\begin{abstract}
One of the most important environments in which accretion discs are found occur in interacting binaries. In this chapter I review the main properties of binary systems and the most important types of binary interactions, stable and unstable mass transfer, the role of mass loss, mass accretion and, in the most dramatic case, the merging of the two binary components. I particularly emphasise the evolutionary context in which these interactions occur and illustrate this using numerous examples of different types of binaries of current research interest. These include hot subdwarfs, symbiotic binaries, binary supernova progenitors, including the progenitors of Type Ia supernovae and potential progenitors of long-duration gamma-ray bursts, low-, intermediate- and high-mass X-ray binaries, containing both neutron stars and black holes, and their descendants, including binary millisecond pulsars, Thorne-Żytkow objects and short-duration gamma-ray bursts.
\end{abstract}

\subsection{Introduction}

One of the main site for accretion discs are interacting binary systems. Indeed, he majority of stars are found in binary systems and, in many cases (up to $\sim 50 \%$ ), they are close enough that mass flows from one star to the other, in many cases forming an accretion disc. This can happen for a wide variety of different systems, systems containing two normal non-degenerate stars, or one compact star (white dwarf [WD], neutron star [NS] or black hole [BH]), or even two compact stars of various combinations. The purpose of the chapter is to provide an overview over the evolution of binary systems, starting with the fundamentals of binary evolution in $\S 1.2$, followed by a selection of current topics in binary evolution theory in $\S 1.3$

1 Oxford University, Oxford OX1 3RH, UK 
and the effects of binary evolution on the final fate of stars and supernovae in $\S 1.4$. The last two sections $\S 1.5$ and $\S 1.6$ discuss low-/intermediate-mass X-ray binaries and high-mass $\mathrm{X}$-ray binaries, respectively.

\subsection{Fundamentals of Binary Evolution}

\subsubsection{Basic Properties}

Most stars in the sky are in binary systems or, more generally, in multiple systems (triples, quadruples, quintuplets, ...), where the orbital periods $\left(P_{\text {orb }}\right)$ range all the way from $11 \mathrm{~min}$ (for a NS-WD binary) to $\sim 10^{6} \mathrm{yr}$. Of course, the majority of binaries are in fairly wide systems that do not interact strongly and where both stars evolve essentially as single stars. But there is a large fraction of systems (with $P_{\text {orb }} \lesssim 10 \mathrm{yr}$ ) that are close enough that mass is transferred from one star to the other which changes the structures of both stars and their subsequent evolution. While the exact numbers are somewhat uncertain, binary surveys suggest that the range of interacting binaries, which are the systems of interest in this chapter, is in the range of $30 \%$ to $50 \%$ (where the binary fraction is higher for more massive stars; see, e.g., Duquennoy \& Mayor 1991; Kobulnicky \& Fryer 2007). A very approximate period distribution, and very useful rule of thumb, is that the distribution in $\log P_{\text {orb }}$ is $\log$ arithmically flat (i.e., $f\left(\log P_{\text {orb }}\right) \simeq$ constant), where each decade of $\log P_{\text {orb }}$ contains $10 \%$ of systems from $10^{-3} \mathrm{yr}$ to $10^{7} \mathrm{yr}$. The massratio distribution (i.e., $q=M_{2} / M_{1}$, where $M_{1}$ and $M_{2}$ are the initially more massive [the primary] and the initially less massive star [the secondary], respectively) is not very well determined but appears to depend somewhat on the mass range. While massive binaries favour stars of comparable mass (i.e., if the primary is a massive star, the secondary is also likely to be relatively massive), this is less clear for low-mass stars; it is sometimes argued that, for low-mass binaries, the masses may be independently chosen from a standard initial mass function [IMF], although most studies show that there is also some bias, possibly consistent with a flat mass-ratio distribution. These differences clearly reflect differences in the formation processes of low- and high-mass stars, that are still extremely poorly understood. Finally, there is generally a large scatter in the distribution of eccentricities $e \equiv \sqrt{1-b^{2} / a^{2}}$, where $a$ and $b$ are the semi-major and semi-minor axis, respectively. Close binaries (with $P_{\text {orb }}<10 \mathrm{~d}$ ) tend to be circular, but this is the result of tidal interactions that efficiently circularise close eccentric binaries. 


\subsubsection{Observational Classification}

One of the main classifications of different types of binary systems is how they appear to an observer. Visual Binaries are systems where the periodic motion of both components can be seen in the sky (see, e.g., Sirius A and B). If the motion of only one star is observable, the binary is referred to as an Astrometric Binary.

Spectroscopic Binaries are systems where the periodic Doppler shifts (due to the orbital motion of the binary components) can be detected in one or more spectral lines. Depending on whether these Doppler shifts can be measured for just one or both binary components, these systems are called single-lined or double-lined spectroscopic binaries.

Photometric Binaries are systems where one can observe a periodic variation of the flux or colour, etc., of the system. However, this does not necessarily prove the binary nature of a system, as variable stars (e.g., Cepheids, RR Lyrae variables) can show similar periodic variations.

Finally, if at least one star eclipses the other star during part of the orbit, the system is an eclipsing binary, which play a particularly important role in determining basic stellar parameters of stars (such as radius and mass).

\subsubsection{The Binary Mass Function}

Another important concept helping to constrain the masses of the components in a binary is the binary mass function. By equating the gravitational force to the centripetal force of either of the components, using various Newtonian relations and assuming a circular orbit, it is easy to derive the following two relations, referred to as the mass functions for stars 1 and $2, f_{1}$ and $f_{2}$,

$$
f_{1}\left(M_{2}\right)=\frac{M_{2}^{3} \sin ^{3} i}{\left(M_{1}+M_{2}\right)^{2}}=\frac{P\left(v_{1} \sin i\right)^{3}}{2 \pi G}
$$

and

$$
f_{2}\left(M_{1}\right)=\frac{M_{1}^{3} \sin ^{3} i}{\left(M_{1}+M_{2}\right)^{2}}=\frac{P\left(v_{2} \sin i\right)^{3}}{2 \pi G} .
$$

Note that the expressions on the right-hand sides of these equations only contain measurable quantities, such as the projected radial velocity amplitudes of the two components $\left(v_{1 / 2} \sin i\right.$, where $i$ is the orbital inclination of the binary) and the orbital period $P$ ( $G$ is the gravitational constant). The terms in the middle contain, besides $\sin i$, the main quantities of interest, the masses of the two components, $M_{1}$ and $M_{2}$.

Thus, the mass functions directly relate the two masses to observable quantities. For a double-lined spectroscopic binary, where the radial velocity amplitudes 
of both components can be measured, one can use these relations to determine $M_{1} \sin ^{3} i$ and $M_{2} \sin ^{3} i$. It is often difficult to determine the inclination; but in cases where this is possible (e.g., for an eclipsing binary with $i \simeq 90^{\circ}$ or for a visual binary), one obtains the masses of both components. Indeed, this is one of the most important methods for determining stellar masses, including those of compact objects, such as black holes. In the case where one star is much less massive than the other (e.g., $M_{1} \ll M_{2}$ ), its mass function directly constrains the mass of the other component since, in this case, equation 1.1 simplifies to $f_{1}\left(M_{2}\right) \simeq M_{2} \sin ^{3} i$.

In the case of doubly eclipsing binaries, one can also determine the radii of both stars. Such systems are the main sources for determining accurate masses and radii of stars, and luminosities, if their distances are also known.

\subsubsection{The Roche Lobe}

One particularly important concept in studying the evolution of binary systems is the Roche lobe. Considering the so-called restricted three-body problem, where one follows the motion of a mass-less test particle in the gravitational field of two orbiting masses $M_{1}$ and $M_{2}$, one can define an effective potential in a co-rotating frame that includes the gravitational potential of the two stars and the centrifugal force acting on the test particle (this assumes that the orbit is circular and that the Coriolis force can be neglected, at least initially). This potential has 5 so-called Lagrangian points where the gradient of the effective potential is zero (i.e., where there is no force in the co-rotating frame). The three most important ones lie along the line that connects the two stars. Of particular importance is the inner one, referred to as $\mathrm{L}_{1}$ or inner Lagrangian point, since the equipotential surface that passes through this point (called the Critical Roche-Lobe Potential) connects the gravitational spheres of influence of the two stars. This means that, if one star starts to fill its Roche lobe (the part of the critical potential engulfing the star), then matter can flow through the $\mathrm{L}_{1}$ point into the Roche lobe of the other star. This is the most important way of how mass can be transferred from one star to the other and is called Roche-lobe overflow (RLOF).

The effective Roche-lobe radius $R_{\mathrm{L}}$ only depends on the orbital separation $A$ and the mass-ratio $q$. For star ' 1 ' with mass $M_{1}$, it is well approximated by

$$
R_{\mathrm{L}}=\frac{0.49 q^{-2 / 3}}{0.6 q^{-2 / 3}+\ln \left(1+q^{-1 / 3}\right)} A
$$

(Eggleton 1983), where $q \equiv M_{2} / M_{1}$ (and an analogous expression for the effective Roche-lobe radius of star ' 2 ').

Another useful way of classifying binaries is how the actual radii of the two stars compare to their respective Roche-lobe radii. In detached binaries, both stars un- 
derfill their respective Roche lobes (i.e., have radii smaller than their Roche-lobe radii). In this case, no mass transfer via RLOF can take place, and the main gravitational interaction occurs via mutual tides (as, e.g., in the Earth-Moon system). In cases where one star has a very strong stellar wind (e.g., for relatively massive stars), a fraction of this wind may be gravitationally accreted by its companion. This alternative, but generally much less efficient type of mass transfer is referred to as wind mass transfer and can also sometimes by important (e.g., in the case of high-mass X-ray binaries; see $\S 1.6$ ).

If one star fills its Roche lobe, the binary is called a semi-detached binary; these are the systems where mass transfer takes place via RLOF.

Finally, it is also possible that both stars fill or even overfill their Roche lobes. In this case, a common photosphere forms that engulfs both components. These system are called either Contact Binaries (observationally referred to as W Ursae Majoris stars) or Common-Envelope Binaries. For such systems, the other two Lagrangian points, $\mathrm{L}_{2}$ and $\mathrm{L}_{3}$, that lie along the axis connecting the two stars but outside their orbit, can become important: if the common envelope reaches either of these two points, then mass can flow through it from the binary to the outside and possibly lead to the formation of a circumbinary disc surrounding the whole binary system.

\subsubsection{Types of Binary Interactions}

While most stars in the sky are probably in binary system, the only ones we are interested here are those where at least one of the components transfers mass to the other one by RLOF (typically $30-50 \%$ of all systems). For the first phase ${ }^{2}$ of mass transfer for one of the stars, one distinguishes three cases of mass transfer depending on the nuclear evolutionary state of the star: Case $A$ (the star is on the main sequence burning hydrogen), Case $B$ (the star has finished hydrogen burning, but not helium burning in the core), Case $C$ (the star has completed core helium burning). Figure 1.1 shows the radius evolution of a $5 M_{\odot}$ star as a function of time and indicates the range where the different cases occur. Since the radius of the star expands only very little (a factor of $\sim 2$ ) on the main sequence but a factor of more than 10 before helium ignition and again after helium burning, it is much more likely that RLOF starts after the star has continued its main-sequence phase (this assumes a logarithmically flat initial period distribution). On the other hand, since a star spends most of its life on the main sequence, it follows that most binaries observed in the sky have not yet had a strong binary interaction, but many of them will do so in the future. This is particularly important when studying the end states

2 If a star experiences more than one mass-transfer phase, the nomenclature quickly becomes complicated, and there is no established standard notation. 


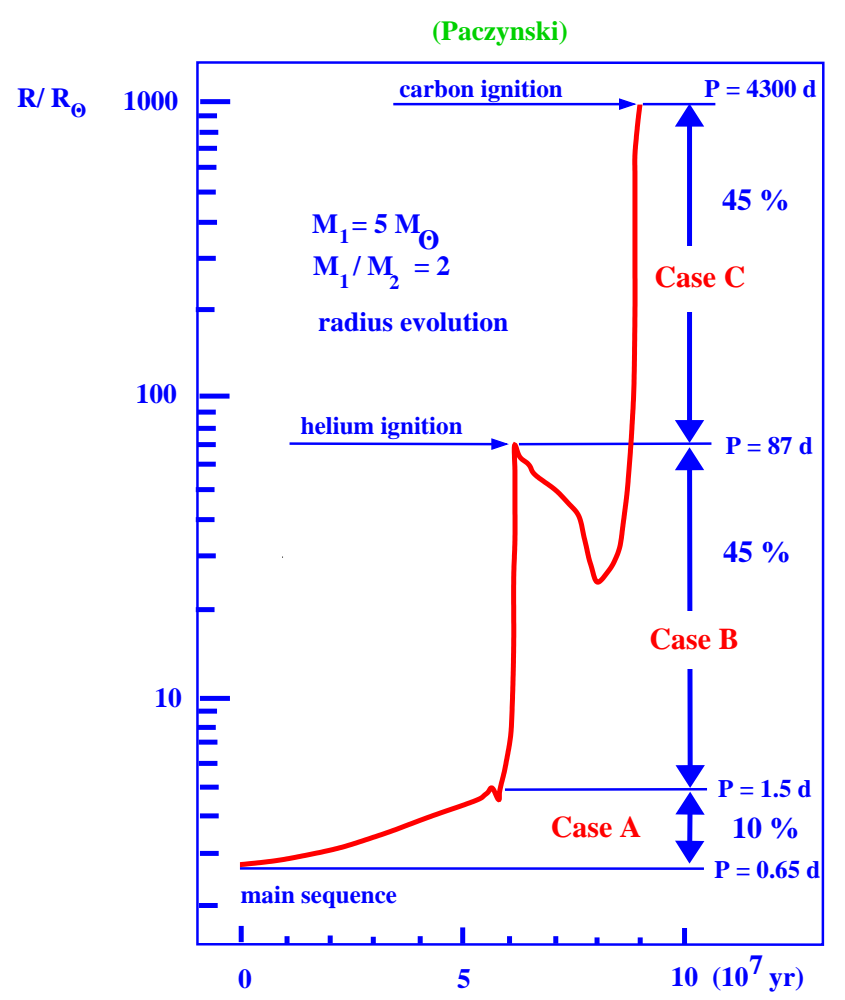

Figure 1.1 The evolution of the radius of a $5 M_{\odot}$ star as a function of its lifetime to illustrate the ranges in radius and orbital period for the different cases of RLOF phases, as indicated, assuming a $2 M_{\odot}$ companion.

of stars and supernovae that probe the late evolutionary phases of a star (see $\S 1.4$ ). Note also that quite massive stars $\left(\gtrsim 20 M_{\odot}\right)$ tend to expand only moderately after helium core burning, and hence, for massive stars, Case $\mathrm{C}$ mass transfer tends to be much less important than Case B mass transfer, where most of the expansion occurs.

When RLOF occurs, one has to distinguish between different modes of mass transfer, depending on whether mass transfer is stable or unstable with very different outcomes.

\section{Stable mass transfer}

Stable, (quasi-)conservative mass transfer (as illustrated in Fig. 1.2) is the easiest type of mass transfer to understand. In this case, most, but not necessarily all, of the transferred mass is accreted by the companion star, generally leading to a widening of the binary. Mass transfer ends when most of the hydrogen-rich envelope of 


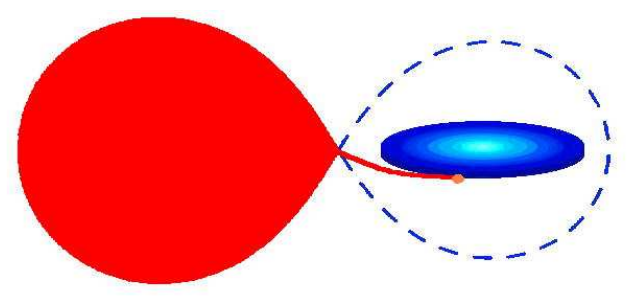

Figure 1.2 Cartoon illustrating stable mass transfer.

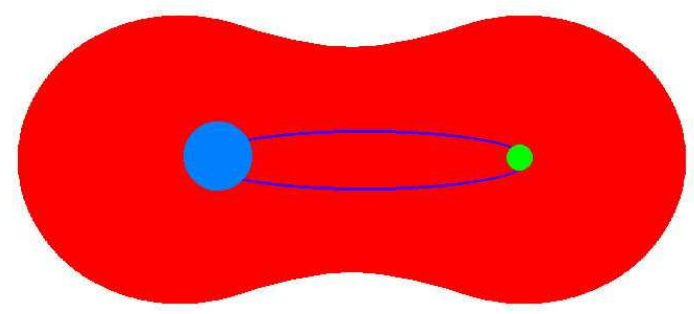

Figure 1.3 Cartoon illustrating unstable mass transfer.

the donor star has either been transferred to the companion or been lost from the system. The end product will be a hydrogen-exhausted helium star with at most a small hydrogen-rich envelope. ${ }^{3}$ Mass accretion will also change the structure of the accreting star. If it is still on the main sequence, the accretor tends to be rejuvenated and then behave like a more massive normal main-sequence star. On the other hand, if it has already left the main sequence, its evolution can be drastically altered, and the star may never evolve to become a red supergiant, but explode as a blue supergiant (if it is a massive star; Podsiadlowski \& Joss 1989).

\section{Unstable mass transfer and common-envelope evolution}

Mass transfer is unstable when the accreting star cannot accrete all off the material transferred from the donor star. The transferred material than piles up on the accretor and starts to expand, ultimately filling and overfilling the accretor's Roche lobe. This leads to the formation of a common-envelope (CE) system, where the core of the donor and the companion form a binary immersed in the envelope of the donor star (see Fig. 1.3). This typically happens when the donor star is a giant or supergiant with a convective envelope, since a star with a convective envelope tends to expand rather than shrink when it loses mass very rapidly (adiabatically), while the Roche-lobe radius shrinks when mass is transferred from a more massive to a less massive star; this makes the donor overfill its Roche lobe by an ever larger amount

3 Stable mass transfer can also occur for an expanding hydrogen-exhausted helium star (so-called Case BB mass transfer). In this case, the star is likely to lose a large fraction/most of its helium envelope. 
and causes runaway mass transfer on a dynamical timescale (so-called dynamical mass transfer).

Once a CE system has formed, friction between the immersed binary and the envelope will make the two components spiral towards each other until enough orbital energy has been released to eject the envelope (Paczyński 1976). This ends the spiral-in phase and leaves a much closer binary with an orbital period typically between $\sim 0.1$ and $\sim 10 \mathrm{~d}$, consisting of the core of the giant and a normal-star secondary. In contrast to the stable RLOF channel, CE evolution tends to produce very short-period systems. Indeed, this is believed to be the main mechanism by which an initially wide binary, with an orbital period of possibly many years, can be transformed into a very close binary with an orbital period of hours to days (Paczyński 1976). Since this spiral-in phase is very short-lived, the immersed companion star will not be able to accrete much matter and will immerse little changed from the CE phase.

\section{Binary mergers}

The most dramatic consequence of a CE phase is that the orbital energy that is released in the spiral-in phase in not sufficient to eject the envelope. In this case, the spiral-in process continues till the core of the donor has merged with the companion, producing a single, initially rapidly rotating star (such as FK Comae stars).

Binary mergers are one of the least studied phases of binary evolution. Despite of their lack of attention, binary mergers are by no means rare events: estimates based on binary population synthesis (BPS) studies suggest that $\sim 5-10 \%$ of all stars experience a complete merger with a companion star during their evolution. Such binary mergers are likely to be responsible for many eruptive events in the Galaxy (e.g., V838 Mon; Tylenda \& Soker 2006).

\subsubsection{Mass-Transfer Driving Mechanisms}

In order to have mass transfer, at least one of the stars has to fill and to continue to fill its Roche lobe. There are two fundamentally different modes of how this is achieved: one is that the donor star tries to expand because of its own internal evolution (this can occur either on a nuclear or a thermal timescale); the other is that the binary system loses angular momentum, causing a shrinking of the orbit.

\section{Expansion of the donor}

The simplest driving mechanism is the expansion of the donor star due to its own nuclear evolution. If such a donor transfers mass to a more massive companion, mass transfer will take place on a nuclear timescale, and the mass-transfer rate $\dot{M}$ will be of order $M / t_{\text {nuc }}$, where $M$ is the mass of the donor star and $t_{\text {nuc }}$ its nuclear timescale. 
However, the situation already becomes more complicated if the donor star is initially more massive than the accretor as, in this case, the donor's Roche lobe will shrink initially. To illustrate this, let us consider the case of conservative mass transfer, where all the mass lost by the donor is accreted by the companion, and the total angular momentum of the binary is conserved. The total angular momentum, $J$, of a binary can be written as

$$
J=\frac{M_{1} M_{2}}{M_{1}+M_{2}} \sqrt{G\left(M_{1}+M_{2}\right) A} .
$$

If $J$ and $M_{1}+M_{2}$ are constant (conserved), this immediately implies that

$$
\left(M_{1} M_{2}\right)^{2} A=\text { constant . }
$$

when $M_{1}=M_{2}$. This implies that, for $M_{1}=M_{2}$, the orbital separation $(A)$ has a minimum. So, if initially $M_{1}>M_{2}$ (assuming star 1 is the donor star), as star 1 transfers mass to star 2 and the masses become more equal, the orbital separation necessarily shrinks. Since the Roche-lobe radius scales roughly with the separation (with a relatively weak mass dependence; eq. 1.3), this also implies that the Roche-lobe radius of star 1 becomes smaller and that star 1 has to shrink to be just able to fill its Roche lobe. Since this radius will generally be smaller than the star's thermal equilibrium radius, this means that it can no longer remain in thermal equilibrium. On the other hand, a star taken out of thermal equilibrium will try to re-establish thermal equilibrium. In this case, as the equilibrium radius is larger, it will try to expand against the shrinking Roche lobe. This expansion will drive more mass across the Roche lobe, indeed generally becomes the main masstransfer driving mechanism. Since the expansion occurs on the thermal timescale of the star, $\dot{M}$ can very roughly be estimated as $M_{1} / t_{\mathrm{KH}}$, where $t_{\mathrm{KH}}$ is the thermal (Kelvin-Helmholtz) timescale of the star. This mode of mass transfer is referred to as thermal timescale mass transfer.

Once the two masses have equalised and $M_{1}<M_{2}$, the orbital separation and the donor's Roche lobe start to increase and, after another thermal timescale or so, the donor will be able to re-establish thermal equilibrium, and subsequently mass transfer will occur on its nuclear timescale (nuclear driven mass transfer). Note that the thermal timescale is generally orders of magnitude shorter than the nuclear timescale. This implies that the mass-transfer rate in the initial phase (with $M_{1}>M_{2}$ ) will be orders of magnitude larger than in the later nuclear driven phase and that this early phase of rapid mass transfer will be concomitantly shorter than the later slow mass-transfer phase. This also means that, when one sees a masstransferring binary, one is always more likely to observe it in the later slow phase.

\section{Angular momentum loss from the system}

There are two main causes of angular momentum loss in a binary that cause a 
shrinking of the orbit and can drive mass transfer: gravitational radiation and magnetic braking. ${ }^{4}$

Two masses orbiting each other cause a periodic distortion of the spacetime continuum around them, i.e., generate a gravitational wave. Since a gravitational wave carries both energy and angular momentum, this means that the binary loses angular momentum. This angular-momentum loss is well described by the standard formula, directly derived from Einstein's theory of General Relativity (Landau \& Lifshitz 1959; Faulkner 1971),

$$
\frac{d \ln J_{\mathrm{GR}}}{d t}=-\frac{32}{5} G^{3} c^{5} \frac{M_{1} M_{2}\left(M_{1}+M_{2}\right)}{A^{4}},
$$

where $c$ is the speed of light. Gravitational radiation as a mass-driving mechanism is only important for fairly close systems, with orbital periods $\lesssim 12 \mathrm{hr}$ (depending on the masses of the components).

Magnetic braking, on the other hand, is far less well understand. Any star with a convective envelope (like the Sun) loses angular momentum due to the magnetic coupling of its wind to the rotation of the star. For example, in the case of the Sun, the solar wind is in co-rotation with the Sun's rotation up to about 10 stellar radii. Indeed, the Sun is rotating so slowly because it has been magnetically braked efficiently in its past. The same will be true if such a star is in a binary, except that, for a sufficiently close binary, the rotation of one or both components will be tidally locked to the orbit (i.e., the rotation period is the same as the orbital period). Thus, in this case, the angular momentum carried away in the magnetic wind is ultimately extracted from the binary orbit, not just the rotation of the star, causing the binary system to shrink.

While there is plenty of evidence that magnetic braking is an important angularmomentum loss mechanism in binaries, the details are still quite uncertain. A commonly used formula (Verbunt $\&$ Zwaan 1981) is

$$
\frac{d J_{\mathrm{MB}}}{d t}=-3.8 \times 10^{-30} M_{2} R^{4} \omega^{3} \mathrm{dyn} \mathrm{cm}
$$

where star 2 is the donor star with radius $R$ and $\omega$ is its angular rotation speed, assumed to be synchronised with the orbit. Note that generally magnetic braking is only included for stars that have convective envelopes, as stars with radiative envelopes or fully convective stars may not have a magnetic dynamo to produce a magnetically coupled wind.

\footnotetext{
4 Another mechanism in principle is the spiralling-in of a binary in a common envelope caused by the friction with the envelope.
} 


\subsection{Selected Current Topics in Binary Evolution}

\subsubsection{Key Uncertainties in Modeling Binary Interactions}

Despite the success in modeling a wide variety of different types of binary systems in recent years, there are still major uncertainties in modeling some of the very basic types of binary interactions. In the following, I will discuss some of the major uncertainties.

\section{The common-envelope phase}

Common-envelope (CE) evolution is undoubtedly the least understood binary interaction (see, e.g., Iben \& Livio 1993; Taam \& Sandquist 2000; Podsiadlowski 2001). It typically involves the spiral-in of a companion star inside the envelope of a (super-)giant donor star and, in many cases, the ejection of the envelope, transforming an initially wide binary into a very close binary (Paczyński 1976). Most typically, it occurs when the radius of the mass-losing star expands more rapidly than the radius of its Roche lobe, leading to dynamical mass transfer. The conditions for the occurrence of dynamical mass transfer are not very well determined. In BPS simulations, it is still occasionally assumed that mass transfer from a star with a convective envelope is dynamically unstable if the mass ratio $q$ of the mass donor to the mass accretor is larger than a critical value $\simeq 0.7$ (this is the appropriate value for a fully convective polytropic star). However, this does not take into account the stabilising effect of the compact core of the giant (e.g., Hjellming \& Webbink 1987), and indeed full binary evolution calculations show that a much more typical critical mass ratio is $1.2(1.1-1.3), 70 \%$ larger than the commonly used value (see, e.g., Han et al. 2002). Indeed, there is observational evidence, e.g., from the orbital-period distribution of symbiotic binaries, that a common-envelope phase may lead to drastic mass loss from the system, but without being accompanied by a dramatic spiral-in phase (see $\S 1.3 .2$ ).

One of the biggest uncertainties in modeling CE evolution is the condition that leads to CE ejection. The most commonly used criterion is that the $\mathrm{CE}$ is ejected when the orbital energy times some efficiency factor $\alpha_{\mathrm{CE}}$ exceeds the binding energy of the envelope; but this simple formula involves numerous uncertainties, in particular, whether the binding energy is estimated from a simple analytic expression or from realistic envelope structures obtained from calculated stellar models (e.g., Dewi \& Tauris 2000) and whether the ionisation energy should be included in the energy balance (see Han et al. 2002 for discussions). The simplistic application of such a criterion can also lead to the violation of energy conservation (by up to a factor of 10 in some published studies). Moreover, in cases where the spiral-in becomes self-regulated and where all the energy released in the spiral-in can be radiated away at the surface of the common envelope (Meyer \& Meyer-Hofmeister 
1979; Podsiadlowski 2001), an energy criterion is no longer appropriate. Finally, it is also not clear whether this treatment is applicable to CE phases where the donor star has a radiative envelope (as may happen when a star starts to fill its Roche lobe in the Hertzsprung gap). Indeed, it seems more likely that this leads to a frictionally driven wind, at least initially, rather than a classical CE phase (Podsiadlowski 2001).

\section{Non-conservative mass transfer}

Another major uncertainty in modeling binary evolution is the treatment of nonconservative mass transfer, in particular the amount of specific angular momentum that is lost from the system. Different reasonable prescriptions can give very different evolutionary paths. Depending on how angular momentum is lost from the system, mass transfer can either be stabilising or de-stabilising. Various studies on classes of particular binaries have shown that mass transfer must often be very nonconservative: these include classical Algols (van Rensbergen, de Loore \& Jansen 2006) and sdB binaries with white-dwarf companions; see $\S$ 1.3.2).

\subsubsection{Hot Subdwarfs}

Hot subdwarfs, or sdB stars, are helium-core-burning stars, typically with a mass $M_{\mathrm{sdB}} \simeq 0.5 M_{\odot}$, that have lost almost all of their hydrogen-rich envelopes by mass transfer in a binary system (for detailed recent studies, see Han et al. [2002, 2003]). In order for the mass donor to be able to ignite helium, the progenitor of the sdB star typically has to fill its Roche lobe near the tip of the first red-giant branch. This can occur either through stable RLOF or in a CE phase. Alternatively, a hot subdwarf can be produced by the merger of two helium white dwarfs if helium is ignited in the merger product (see Fig. 1.4 for an overview of the various channels). This single class of binary system therefore on its own illustrates a large variety of the different types of binary interactions involving a compact component. Since the evolutionary history of sdB stars is so well defined, they are particularly suitable for testing and constraining binary evolution theory. The studies by Han et al. $(2002,2003)$ have been very successful in reproducing the main observed properties of these systems. There main conclusions were: (1) the three major formation channels, involving (a) stable RLOF, (b) CE evolution and (c) binary mergers are expected to be of comparable importance; (2) the orbital period distribution of short-period sdB binaries (with orbital periods $\lesssim 10 \mathrm{~d}$ ) is well reproduced if the CE ejection mechanism is very efficient (with $\alpha_{\mathrm{CE}}>0.75$ ) and a large fraction of the recombination energy can be used in the process; (3) in order to reproduce the short-period sdB binaries with white-dwarf companions (in the second, unsta- 


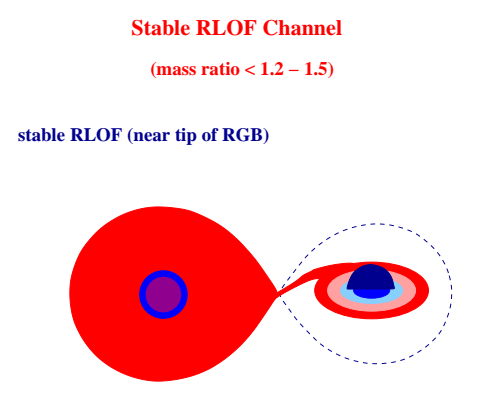

wide SdB binary with MS/SG companion

○

$$
\begin{aligned}
& P_{\text {orb }}=10-500 \text { days } \\
& M_{\text {sdB }}=0.30-0.49 M_{\text {sun }}
\end{aligned}
$$

Common-Envelope Channels

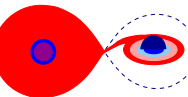

unstable RLOF ---> dynamical mass transfer

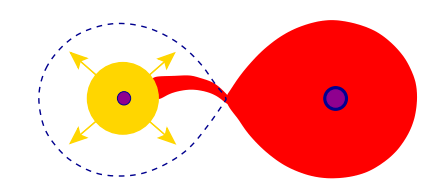

common-envelope phase

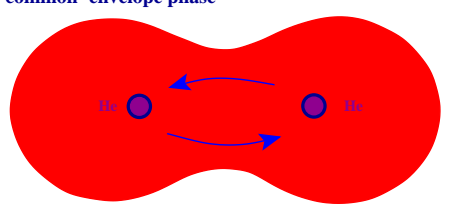

short-period sdB binary with He WD companion

( unstable RLOF ---> dynamical mass transfer

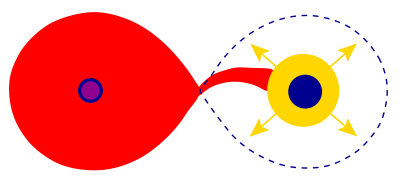

common-envelope phase

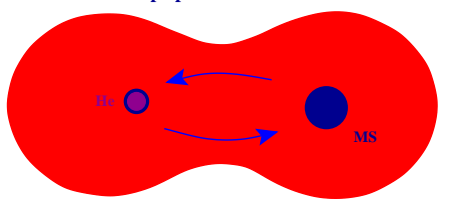

short-period sdB binary with MS companion

○

Figure 1.4 Binary channels illustrating the formation of hot subdwarfs. Top panel: Stable Roche-lobe overflow. Bottom panel: CE channels. In addition, a hot subdwarf may form from the merger of two He white dwarfs (or a He and $\mathrm{HeCO}$ white dwarf). 
ble mass-transfer phase; the bottom left channel in Fig. 1.4), the first mass-transfer phase has to be stable and very non-conservative.

Since hot subdwarfs are the dominant source of UV light from an old population in our Galaxy, it is only natural to assume that hot subdwarfs in binaries are also an important source of UV light in other old populations, such as early-type galaxies. Indeed, Han, Podsiadlowski \& Lynas-Gray (2007) have shown that the same binary model that is so successful in our own Galaxy, when applied to early-type galaxies, can reproduce the UV upturn, a long-standing puzzle in the field, without any ad hoc assumptions. It also predicts that the UV upturn should develop after an age of the population of $\sim 1 \mathrm{Gyr}$ and only show a fairly weak metallicity dependence (although the latter point is still under investigation). This example illustrates particularly well how important it is to understand the complexities of stellar populations in our Galaxy before one can trust the modeling of stellar populations in other galaxies. It would be unreasonable to assume that the stellar populations in external galaxies are much simpler than in the Milky Way, a mistake that is, however, still commonly made.

\subsubsection{Binary Mergers}

Binary mergers are another topic of major current interest, in particular, since present and future transient surveys are likely to detect such mergers in real time (if they have not been done so already; see the case of the optical transient in M85; Kulkarni et al. 2007). Since a large fraction of the orbital binding energy is released in a merger, the merger process itself is expected to resemble a faint supenova ("supernova impostor"), such as the outburst of eta Carinae in the 19th century. After the merger, the remnant will be a rapidly rotating supergiant, at least initially rotating near break-up at the equator. This is probably the major channel for producing $\mathrm{B}[\mathrm{e}]$ supergiants, which are evolved stars rotating new breakup which probably cannot be formed via any other 'reasonable' single-star channel (see Podsiadlowski, Morris \& Ivanova 2006 for further discussions and details).

In general, binary mergers can occur on a large range of timescales. This depends mainly on the structure of the envelope, in particular the density profile, since this determines the friction timescale and hence the spiral-in timescale during the spiral-in phase. This immediately implies that different merger types have to be distinguished depending on whether the envelope is initially convective or radiative.

In the case of radiative envelopes, very little mass is contained in the outer lowdensity envelope (typically less than $1 \%$ of the total mass in the outer $50 \%$ or the radius of the star), but there is a fairly steep density gradient (see Fig. 2 of Podsiadlowski 2001). This implies that the frictional luminosity, which is proportional 
to the density, is initially very low, and that there is a long initial "contact" phase without significant spiral-in. This phase may last 100s to 1000s of years. Even a moderate shrinking of the orbit releases enough orbital energy to eject most of the mass surrounding the binary in a frictionally driven wind. If the mass ratio is sufficiently close to 1 , this CE phase may be temporary, and the system may survive as a semi-detached binary. However, once the immersed companion reaches the high-density layers of the envelope, the spiral-in accelerates and always runs away, ultimately occurring on a dynamical timescale (because of the steep density gradient, envelope expansion can never lead to a self-regulated spiral-in as in the case of convective envelopes).

In contrast, in a convective envelope most of the mass of the envelope is contained in the outer parts of the envelope. Hence a convective envelope tends to have a much higher density in the outer parts, but a much shallower density gradient (see Fig. 2 of Podsiadlowski 2001). This implies that the initial spiral-in (after the loss of co-rotation) is much faster (because of the higher density), but once the envelope has expanded sufficiently the spiral-in can become self-regulated, where the frictional luminosity is low enough to be completely radiated away at the surface rather than drive further expansion of the envelope (Meyer \& Meyer-Hofmeister 1979). The typical timescale for the spiral-in and merging phase in this case is 100s of years, and we refer to such mergers as slow mergers rather than dynamical mergers. A third type of merger can occur in dense clusters, where stars can collide directly to merge in collisional mergers.

\section{A Slow Merger Model for SN 1987A}

Probably one of the most interesting cases of a binary merger is the progenitor of SN 1987A for which a merger model provides the most likely explanation for the unusual properties of this supernova (Podsiadlowski, Morris \& Ivanova 2007). However, after the two components in a binary have merged, apart from rapid rotation, there is little direct evidence that the newly formed object once was a binary system. Arguably the best evidence for the former binary nature of the progenitor of SN 1987A stems from the spectacular triple-ring nebula surrounding the supernova, first discovered with the NTT (Wampler et al. 1990) and best imaged with the HST (Burrows et al. 1995). All of the material in the ring nebula was ejected from the progenitor system some $\sim 20,000 \mathrm{yr}$ before the explosion and provides a unique fingerprint of the dramatic events that occurred at that time (see the model by Morris \& Podsiadlowski 2007). Its almost axi-symmetric, but very non-spherical structure suggests rotation as an important physical ingredient, but elementary angular-momentum considerations imply that any single massive star that rotated rapidly on the main sequence could not possibly be rotating sufficiently 
rapidly as a red supergiant to produce the observed asymmetries. A source of angular momentum is required, most likely in the form of orbital angular momentum that was converted into spin angular momentum during a merger event some $\sim 20,000 \mathrm{yr}$ before the explosion.

\section{The B[e] Supergiant $\mathrm{R} 4$}

The B[e] supergiant R4 in the Small Magellanic Cloud provides perhaps the most convincing evidence that at least some $\mathrm{B}[\mathrm{e}]$ supergiants are the results of binary mergers. The $\mathrm{B}[\mathrm{e}]$ component has a luminosity of $L \simeq 10^{5} L_{\odot}$ and effective temperature $T_{\text {eff }} \simeq 27,000 \mathrm{~K}$, and its mass has been estimated to be $12 M_{\odot}$. The $\mathrm{B}[\mathrm{e}]$ supergiant is a member of a binary system with an orbital period of $21.3 \mathrm{yr}$ and the companion is an evolved A supergiant (Zickgraf et al. 1996). Moreover, NTT spectra suggest that the system is surrounded by a "cloverleaf" or double bi-polar nebula with a characteristic expansion velocity of $v \sim 100 \mathrm{~km} \mathrm{~s}^{-1}$ and a size of $2.4 \mathrm{pc}$, giving the nebula a dynamical age of $10^{4} \mathrm{yr}$ (Pasquali et al. 2000). The composition of the nebula is also enriched in nitrogen, suggesting that $\mathrm{CNO}$ processed material has been ejected.

The most puzzling feature of the system is, however, the fact that the more evolved A supergiant is much less luminous (roughly by a factor of 10) than the $\mathrm{B}[\mathrm{e}]$ supergiant, the opposite of what one would expect if the two stars formed at the same time and evolved in isolation. The likely resolution of this Algol-type paradox (Pasquali et al. 2000) is that the system originally consisted of 3 stars, where the initially most massive component (now the A supergiant) evolved independently from the other two, but where the other two merged after the second most massive component evolved off the main sequence, producing a new object that is more massive and hence more luminous than the original primary, but is still less evolved. The "cloverleaf" nebula could be a combination of an equatorial outflow, associated with an outflow from one of the outer Lagrangian points during the binary contact phase, while the perpendicular structure is the result of a bipolar outflow ejected during the merger phase (plus any subsequent wind interaction).

\section{Eta Carinae}

One of the most spectacular nebulae in our Galaxy is the nebula around $\eta$ Carinae, which was ejected between 1840 and 1860 in a giant outburst, during which the luminosity of the system reached a luminosity of almost $3 \times 10^{7} L_{\odot}$. The mass ejected in the outburst has been estimated to be $\sim 10 M_{\odot}$. Combined with the measured expansion velocities, this gives the nebula a kinetic energy of $\sim 10^{50} \mathrm{erg}$ 
(Smith et al. 2003). This corresponds to about $10 \%$ of the energy released in a typical core-collapse supernova, making this a truly remarkable event.

$\eta$ Car appears to be a member of a relatively wide binary with an orbital period of $5.5 \mathrm{yr}$ in a very eccentric orbit (with an eccentricity $e>0.6$ ), leading to periodic $\mathrm{X}$-ray activity when the companion is near periastron. With this relatively long period, it seems unlikely that the binary companion is responsible for the major outburst. There is also evidence for a latitude-dependent wind suggesting that $\eta$ Car is rapidly rotating.

If it is indeed true that in the major outburst $\sim 10 M_{\odot}$ were ejected with an energy of $\sim 10^{50} \mathrm{erg}$, this would require a very dramatic, dynamical event. Considering that this energy is comparable to the binding energy of a massive early-type supergiant, it seems implausible that this could be caused by an envelope instability, which could at most release the binding energy of the outer envelope, which would be several orders of magnitude less than $10^{50} \mathrm{erg}$.

All of these facts combined again point in the direction of a merger, quite similar to the case of R4, where two components in a triple system merged to produce the outburst in the mid-1800s. This could provide: (1) the energy for the mass ejection; in a dynamical merger, this is expected to be of the order of the orbital energy of the spiralling-in star near the point where it is disrupted. This could easily be as large a $\sim 10^{50} \mathrm{erg}$. (2) Cause the spin-up of the merger product. (3) Provide the excess thermal energy that needs to be radiated away after the merger, driving the post-eruption wind with an inferred wind mass-loss rate of $10^{-3} M_{\odot} \mathrm{yr}^{-1}$.

\subsubsection{Symbiotic Binaries}

\section{A case for quasi-dynamical mass transfer?}

Symbiotic binaries, specifically the so-called S-type symbiotics, which contain a giant donor star transferring mass to typically a white-dwarf companion, provide a major challenge to our current understanding of binary interactions. In particular, the orbital-period distribution $(\sim 100-1400 \mathrm{~d}$; Mikołajewska 2007) cannot be explained by present BPS models that only involve the stable and unstable types of mass transfer discussed in $\S 1.2$. If mass-transfer is unstable, leading to a CE and spiral-in phase, one would expect - even with the most optimistic assumptions about the CE ejection process (Han, Podsiadlowski \& Eggleton 1995) - much shorter orbital periods than the observed ones. In contrast, if mass transfer is stable, this would generally lead to a widening of the systems. In short, standard BPS simulations predict a gap in the orbital-period distribution where most of the S-type symbiotics are actually observed. This problem was first realised by Webbink (1986) and since then there have been a number of proposals to resolve this 
problem. Podsiadlowski et al. (1992) proposed a different mode of mass transfer, quasi-dynamical mass transfer, which has characteristics of both dynamical and stable mass transfer. The basic idea is that, if the mass ratio is relatively close to 1 , the mass-transfer rate is large enough to lead to a common envelope surrounding the binary components (similar to what is seen in contact binaries), but without a significant spiral-in phase. A spiral-in phase is avoided as long as the envelope remains in co-rotation with the binary since, in this case, there is no friction to drive the spiral-in. Typically, the envelope remains tidally locked to the binary if the size of the envelope is less than about twice the orbital separation. During this phase, one expects that most of the mass lost from the giant is ultimately lost from the system mainly through one of the outer Lagrangian points. This may even lead to a circumbinary disc, which itself may tidally couple to the binary and affect the evolution of the binary orbit (e.g., Spruit \& Taam 2001; also see Frankowski \& Jorissen 2007; Dermine et al. [in preparation]). Once the mass ratio has reversed sufficiently, the envelope will recede below the critical Roche potential, and the subsequent evolution will resemble the case of stable, but most likely still very non-conservative mass transfer.

\section{Wind Roche-lobe overflow: a new mode of mass transfer}

Recent observations of the symbiotic binary Mira (o Ceti) have provided another example indicating that our present understanding of binary interactions is incomplete. Mira is a so-called D-type symbiotic where the donor star is a Mira variable in a very wide orbit (in the case of Mira, the orbital period has been estimated to be larger than $\sim 1000 \mathrm{yr}$ ). One would ordinarily not consider such wide binaries as interacting binaries (apart perhaps from some low-level of wind accretion). Nevertheless, X-ray observations by Karovska et al. (2005), which were able to resolve the Mira donor star (Mira A), appear to show that Mira A is filling its Roche lobe. Of course, it cannot be the Mira variable itself, as it is a factor of $\gtrsim 10$ smaller than its Roche lobe, but the slow wind emanating from it. Mira winds are driven by the pulsations of the dynamically unstable Mira envelope, but are only accelerated to their terminal speeds at $\sim 5$ stellar radii, where dust can form and radiation pressure on the dust can provide the necessary acceleration. If this acceleration region is comparable to the radius of the Roche lobe, the wind flow itself will feel the binary potential and can effectively fill the donor star's Roche lobe. The importance of this new type of wind Roche-lobe overflow (WRLOF) is that a large fraction of the wind can be transferred to the companion: the mass-transfer rate may exceed the estimate expected from simple Bondi-Hoyle accretion by up to 2 orders of magnitude. This provides an efficient new mechanism for mass transfer in fairly wide binaries (which we have started to refer to as Case D mass transfer; see Podsiadlowski \& Mohamed 2007). In addition, since any mass that is lost from 
the system is strongly confined to the orbital plane, producing a disc-like outflow (or even circumbinary disc), this is also likely to have important implications for the shaping of asymmetric planetary nebulae.

Case D mass transfer should also be important for massive stars as recent calculations (Yoon \& Cantiello 2010) have shown that massive red supergiants can also develop dynamically unstable envelopes, e.g., experience the 'Mira' phenomenon. Since, in many cases, the consequences of WRLOF are similar to Case $\mathrm{C}$ mass transfer, this may lead to a dramatic expansion of the period range for which such late phases of mass transfer are important. ${ }^{5}$ This may have major implications for various types of supernova progenitors (such as the progenitors of Type II-L, IIb supernovae) and even some binary gamma-ray burst progenitor models, which often require such late phases of mass transfer. At the moment, we are only at the beginning of exploring all the consequences of Case D mass transfer.

\subsection{Late Stellar Evolution and Supernovae in Binaries}

\subsubsection{Major Supernova Explosion Mechanisms}

Generally speaking, a supernova is the explosion of a star. For at least a few decades, however, it has been realised that there are (at least) two main supernova explosion mechanisms: core-collapse supernovae involving the final phase in the evolution of a massive star and thermonuclear explosions, most likely related to white dwarfs approaching the Chandrasekhar limit. ${ }^{6}$

\section{Core-Collapse Supernovae}

The evolution of stars and, in particular, massive stars is characterised by an alternation of nuclear burning phases and contraction phases. For a massive star, the evolution ends when it has developed an iron core, surrounded by onion-like structure consisting of shells of increasingly lower mean atomic mass. Since iron is the most stable nucleus (i.e., has the highest nuclear binding energy per baryon), no more energy can be generated by fusing iron with other nuclei. Therefore, if the core exceeds the Chandrasekhar mass for iron, there is no longer a cold hydrostatic equilibrium configuration, and the core has to contract/collapse as it cools and loses its pressure support. While this contraction may start slowly, it soon accelerates because of a number of instabilities, ultimately reaching free fall. Most of

5 Note that, for rather massive stars, the orbital period range for Case $\mathrm{C}$ mass transfer is very narrow and disappears completely for the most massive stars.

6 The Chandrasekhar limit defines the maximum mass at which a zero-temperature, self-gravitating object can be supported by electron degeneracy pressure. For most compositions of interest, this mass is close to $1.4 M_{\odot}$. 
the gravitational energy that is released in the collapse is ultimately converted into neutrinos which, at least initially, freely escape from the core.

This collapse is only stopped once matter reaches nuclear densities $\left(\rho_{\text {nuc }}\right)$ and the strong force becomes important, providing a sudden repulsive force. Because of the initial over-compression of the matter, now mainly composed of neutrons, the core bounces and drives an outward moving shock into the still infalling outer core. It was once hoped that this shock, which initially carries an energy of $\sim 10^{51} \mathrm{ergs}$ could reverse the infall of the outer core into an outflow and drive a prompt explosion. But because of the continued photo-disintegration of the infalling material, which requires $\sim 10^{51} \mathrm{ergs}$ for $0.1 M_{\odot}$ of $\mathrm{Fe}$, this energy is quickly consumed and the shock stalls; it is now believed that this can never drive a successful prompt explosion.

The total energy that is released in the collapse is of order the binding energy of the neutron star forming at the centre $\left(G M_{\mathrm{NS}}^{2} / R_{\mathrm{NS}} \sim 3 \times 10^{53} \mathrm{ergs} \simeq 0.1 M_{\mathrm{NS}} c^{2}\right.$ for $M_{\mathrm{NS}} \simeq 1.4 M_{\odot}$ and $\left.R_{\mathrm{NS}} \simeq 10 \mathrm{~km}\right)$. This is several orders of magnitude more than the binding energy of the outer core ( $\left.E_{\text {core }} \simeq 10^{51} \mathrm{ergs}\right)$. However, most of this energy escapes freely in the form of neutrinos that only interact weakly with matter. It has remained one of the most enduring unsolved problems in supernova physics, how a fraction $(\sim 1 \%)$ of this energy can be deposited just below the accretion shock and be allowed to accumulate till enough energy is available to drive an explosion. In the presently favoured model of delayed neutrino-driven explosions (e.g., Mezzacappa et al. 2007; Janka et al. 2007), this may require more than $500 \mathrm{~ms}$, which is extremely long compared to the dynamical timescale of the proto-neutron star $(\sim 1 \mathrm{~ms})$. If this mechanism fails, matter will continue to fall onto the proto-neutron star and ultimately convert it into a black hole.

\section{Thermonuclear Explosions}

The second important explosion mechanism has nothing to with massive stars, but is generally believed to occur in accreting $\mathrm{CO}$ white dwarfs when their mass approaches the Chandrasekhar mass. When the CO WD mass reaches $\sim 1.37 M_{\odot}$, carbon is ignited in or near the centre of the white dwarf. Initially this drives convection in the core, transporting the energy outwards and radiating it away in the form of neutrinos (this phase of low-level carbon burning, referred to as the simmering phase, can last for up to $\sim 10^{3} \mathrm{yr}$ ). But, there comes a point when the core is unable to rid itself of the excess nuclear energy, and the burning process becomes explosive. The reason for this nuclear runaway is that the core material is highly degenerate. This means that the core pressure is independent of temperature. Therefore, a rise in central temperature (due to the carbon burning) does not produce an increase in pressure which would limit the increase in temperature 
(the valve mechanism that keeps burning in ordinary stars, supported by thermal pressure, stable). The further increase in temperature increases the nuclear burning further, producing a runaway process which incinerates a large fraction of the white dwarf and ultimately destroys it completely. In the case of a thermonuclear explosion, unlike the case of core collapse, no remnant is expected, and the energy source is purely nuclear energy ( $\left.\sim 10^{51} \mathrm{ergs}\right)$. The fact that the energy in the two types of explosion is comparable ( $\left.\sim 10^{51} \mathrm{ergs}\right)$ is not a coincidence, since, in both cases, the energy scale is set by the binding energy of the core (the CO core in the case of the thermonuclear explosion, and the binding energy of the outer Fe core in the core-collapse case); they are comparable as both are ultimately determined by the same physics of electron degeneracy, which determines the immediate presupernova structure.

In the ensuing explosion, a large fraction of the white dwarf is burned, in the inner part completely to nuclear statistical equilibrium (NSE), which means mainly to iron-group elements, mostly ${ }^{56} \mathrm{Ni}$, and incompletely further out, producing mainly intermediate-mass elements, such as ${ }^{28} \mathrm{Si}$ and ${ }^{32} \mathrm{~S}$. The radioactive ${ }^{56} \mathrm{Ni}$ will subsequently decay to ${ }^{56} \mathrm{Co}$ (with a half life of $7 \mathrm{~d}$ ), powering the supernova lightcurve, and ultimately to ${ }^{56} \mathrm{Fe}$ (with a half life of $77 \mathrm{~d}$ ). A typical supernova of this type produces $\sim 0.7 M_{\odot}$ of ${ }^{56} \mathrm{Fe}$; hence these supernovae are believed to be the dominant producers of iron in the Universe. Since most of them produce very similar amounts of radioactive ${ }^{56} \mathrm{Ni}$, the resulting supernova lightcurves are quite similar, which means that they can be used as standard cosmological distance candles (strictly speaking 'standardisable' distance candles).

Unlike core-collapse supernovae, the physics of thermonuclear explosions is reasonably well understood. One of the lingering uncertainties is how the carbon burning front, which starts as a deflagration (i.e., a sub-sonic burning front), is accelerated into a detonation (i.e., a super-sonic burning front), which seems to be favoured by observations for the majority of thermonuclear explosions.

The main uncertainty, even controversy, is the question of their progenitors, the type of stellar systems in which a $\mathrm{CO}$ white dwarf can grow towards the Chandrasekhar mass. I will return to this issue in more detail later.

\subsubsection{Supernova Classification}

The basic classification of supernovae is quite simple: they are classified as Type I or Type II supernovae, depending on whether they have hydrogen lines in the spectrum (Type II) or lack hydrogen lines (Type I). For a long time, it was thought that these two observational classes may have a one-to-one relation to the two explosion mechanisms discussed in the last section, core collapse supernovae (Type II) and thermonuclear explosions (Type I), respectively. However, over the last three 
decades is has become clear that this is not the case and that, in principle, both explosion types could come in both observational varieties. As a consequence, the basic classification has become much more complex, requiring the introduction of more and more sub-types.

\section{Main Classification Scheme}

The thermonuclear explosion of a $\mathrm{CO}$ white dwarf is now believed to be associated with a Type Ia supernova (SN Ia). These supernovae have no hydrogen, but strong $\mathrm{Si}$ lines. Si and also $\mathrm{S}$ are intermediate-mass nuclei which are produced in abundance in the part of an exploding white dwarf that does not burn completely to NSE; hence, this provides a very characteristic signature for a thermonuclear explosion.

In addition to SNe Ia, there are two other sub-types of Type I supernovae, Type Ib and Type Ic. These types are also defined on the basis of their spectroscopic characteristics, both lack hydrogen, but Type Ib supernovae ( $\mathrm{SNe} \mathrm{Ib}$ ) show He lines, while Type Ic supernovae (SNe Ic) lack both $\mathrm{H}$ and He lines. Unlike SNe Ia, they produce fairly little ${ }^{56} \mathrm{Ni}$ and are found predominantly in or near star-forming region and are therefore believed to be connected with core-collapse supernovae, i.e., the explosion of massive stars that have lost their H-rich envelopes and, in the case of SNe Ic, their He-rich layers as well. ${ }^{7}$

There are also several different sub-types of Type II supernovae. Unlike SNe I, they are not defined by their spectroscopic properties but by their lightcurves, i.e., their luminosity, measured in a particular waveband (typically $B$ or $V$ ) as a function of time. The lightcurves of Type II-P supernovae (SNe II-P), where the 'P' stands for "plateau", show a long phase, lasting up to $\sim 100 \mathrm{~d}$, where the lightcurve is constant (the plateau phase). Their progenitors are most likely massive red supergiants (with a typical mass $\lesssim 20 M_{\odot}$ ) that experience core collapse. The second, much less common variety, Type II-L supernovae (SNe II-L), do not show this plateau but their luminosity drops off more or less linearly (on a logarithmic scale) after the lightcurve peak (hence the letter ' $L$ ' for "linear"). These are almost certainly also core-collapse supernovae, but in this case, the progenitors must have already lost a large fraction of their H-rich envelopes.

\footnotetext{
7 It is presently not entirely clear how much He could be present in a SN Ic. Since He is generally non-thermally excited, it requires the presence of a source of energetic photons, e.g., from the radioactive decay of ${ }^{56} \mathrm{Ni}$. If the He layer is shielded from this radioactive source, it is possible in principle to hide significant amounts of He.
} 


\section{Complications}

Unfortunately, there are many complications going beyond this simple scheme. The progenitor of supernova 1987A (SN 87A) had a large H-rich envelope, but did not have an extended plateau phase, and therefore SN 87A defines a new class of its own. Other supernovae appear to change their type. Supernova 1993J initially looked like a type II supernova, but soon transformed into a supernova resembling a SN Ib. As a consequence, this supernova type is now referred to as a Type IIb supernova (SN IIb). Other sub-types are not directly related to a particular supernova mechanism, but to a supernova-related phenomenon. For example, Type IIn supernovae stand for supernovae that show narrow $\mathrm{H}$ lines $(\mathrm{H} \alpha)$ in emission. This must come from $\mathrm{H}$-rich material in the immediate neighbourhood of the supernova, most likely ejected by the progenitor in the not-too-distant past, that was flash-ionised by the first light from the supernova. This is not necessarily related to a particular explosion type; it just implies a particular mass-loss history of the progenitor. In a more extreme version, there may be so much material around the exploding star that the supernova ejecta are rapidly slowed down by the interaction with this material, converting kinetic energy into thermal energy and ultimately radiation. In this case, the lightcurve shape itself is determined by this interaction with the circumstellar material. Supernovae that show evidence for such interactions are sometimes referred to as Type IIa supernovae (SNe IIa), though how this fits into the overall supernova scheme and, in particular its relation to $\mathrm{SNe} \mathrm{IIb}$, lacks any obvious logic.

Indeed, as this previous discussion shows, the supernova classification scheme has become too complicated and convoluted to be very useful. In fact, sometimes even supernova experts get confused. The problem is that the main scheme is a discrete one, while the supernova properties clearly vary in a continuous manner. Indeed a lot of the diversity of supernova sub-types can be understood as a sequence of increased mass loss

Thus, the whole sequence

$$
\mathrm{SN} \text { II-P } \rightarrow \text { SN II-L } \rightarrow \text { SN IIb } \rightarrow \text { SN Ib } \rightarrow \text { SN Ic }
$$

appears to be a sequence of increased envelope loss, first of the H-rich envelope and then the He-rich layer. The immediate physical question is what causes this mass loss. While stellar winds may play an important role in some cases, binary interactions are almost certainly even more important, since a large fraction, if not the majority, of all massive stars are in relatively close binaries where the components can interact directly (e.g., by mass transfer causing mass loss, mass accretion and, in the most extreme case, by the complete merger of the binary components [Podsiadlowski, Joss \& Hsu 1992]). These interactions particularly affect the enve- 
lope properties of the massive progenitors and hence help to determine the shapes of the resulting lightcurves.

\subsubsection{The Progenitors of Type Ia Supernovae}

Type Ia supernovae ( $\mathrm{SNe}$ Ia) have been very successfully used as standardisable distance candles and have provided the first indication for an accelerating Universe (Riess et al. 1998; Perlmutter et al. 1999). Their use as distance candles relies on the empirical fact that SN Ia lightcurves appear to form a one-parameter family and that there is a relationship between the supernova peak luminosity and the lightcurve width, referred to as the 'Phillips relation' (Phillips 1993), which can be used to infer the peak luminosity and hence the distance. In recent years there has been increasing evidence that not all SNe Ia obey this relation and there is even some evidence that a subset of SNe Ia have progenitors with a mass exceeding the Chandrasekhar mass (e.g., Howell et al. 2006). Indeed, the nature of the progenitors of SNe Ia is still controversial, and the link between progenitor models and explosion models is presently one of the weakest points in our understanding of $\mathrm{SNe}$ Ia.

There is now broad agreement that most SNe Ia are caused by a thermonuclear explosion of a $\mathrm{CO}$ white dwarf when its mass approaches the Chandrasekhar mass. At this point, carbon is ignited in the electron-degenerate core. This causes a thermonuclear runaway, leading to the incineration of a large fraction of the white dwarf and ultimately its complete destruction. Unlike the case of a core-collapse supernova, the energy causing the SN Ia explosion $\left(\sim 10^{51} \mathrm{erg}\right)$ is entirely nuclear energy, and no compact remnant is expected.

What is still uncertain and is indeed controversial is the evolution that produces these Chandrasekhar-mass white dwarfs. The most popular progenitor models fall broadly into two classes, the single-degenerate (SD) model and the doubledegenerate (DD) model.

\section{The Single-Degenerate Model}

In the SD model, the white dwarf grows in mass by accreting from a non-degenerate companion star (Whelan \& Iben 1973; Nomoto 1982), where the companion star can either be a main-sequence star, a helium star, a subgiant or even a giant. The main problem with this class of models is that it is generally difficult to increase the mass of a white dwarf by accretion: if the mass-accretion rate is too low, this causes nova explosions and/or helium flashes (Nomoto 1982) which may eject most of the accreted mass. If the mass-accretion rate is too high, most of the transferred mass must be lost in a disc wind to avoid a merger of the binary, again leading to a low 
accretion efficiency. There is only a very narrow parameter range where a white dwarf can accrete hydrogen-rich material and burn in a stable manner. This parameter range may be increased if differential rotation affects the accretion process (Yoon \& Langer 2004). One promising channel that has been identified in recent years relates them to supersoft X-ray sources (van den Heuvel et al. 1992). However, it is not clear whether this channel produces a sufficient number of systems to explain the observed SN Ia rate in our Galaxy $\left(\sim 3 \times 10^{-3} \mathrm{yr}^{-1}\right.$; Cappellaro $\&$ Turatto 1997; for further discussion see, e.g., Han \& Podsiadlowski 2004; Fedorova, Yungelson \& Tutukov 2004). On the plus side, a number of binary systems are known that are excellent candidates for SN Ia progenitors: U Sco, RS Oph and TCrB all contain white dwarfs that are already close to the Chandrasekhar mass, where the latter two systems are symbiotic binaries containing a giant companion (see Hachisu et al. 1999 for a discussion of this channel). However, in none of these cases is it clear whether the massive white dwarf is a $\mathrm{CO}$ or an ONeMg white dwarf (the latter is not expected to produce a SN Ia).

\section{The Double-Degenerate Model}

In contrast to the SD model, the DD model (Iben \& Tutukov 1984; Webbink 1984) involves the merger of two $\mathrm{CO}$ white dwarfs with a combined mass in excess of the Chandrasekhar mass. This model has the advantage that the theoretically predicted merger rate is quite high (see, e.g., Yungelson et al. 1994; Han et al. 1995; Nelemans et al. 2001), consistent with the observed SN Ia rate and probably the observed number of DD systems discovered by the SPY survey (Napiwotzki et al. 2002). The main problem with this scenario is that it seems more likely that the disruption of the lighter white dwarf and the accretion of its debris onto the more massive one leads to the transformation of the surviving $\mathrm{CO}$ white dwarf into an $\mathrm{ONeMg}$ white dwarf which subsequently collapses to form a neutron star (i.e., undergoes accretion-induced collapse) rather than experiencing a thermonuclear explosion (e.g., Nomoto \& Iben 1985).

Recently, Yoon, Podsiadlowski \& Rosswog (2007) modelled the expected postmerger evolution after a double-degenerate $\mathrm{CO}$ merger. They found that the immediate post-merger product was a low-entropy core, the initially more massive $\mathrm{CO}$ white dwarf, surrounded by a high-entropy envelope and an accretion disc (from the disrupted lower-mass object). Following the thermal and angular-momentum evolution of the merger product, they showed that the evolution is controlled by neutrino losses at the bottom of the envelope and that, despite the very high core accretion rate, carbon ignition could be avoided under some circumstances. More generally, they concluded that the merger could lead to a thermonuclear explosion if two main conditions are satisfied: (1) carbon ignition must be avoided during the 
merging process and (2) the mass accretion rate from the surrounding disc must be less than $\sim 10^{-5} M_{\odot} \mathrm{yr}^{-1}$.

Thus, there may be some parameter range where the conversion into an $\mathrm{ONeMg}$ white dwarf can be avoided (also see Piersanti et al. 2003). This raises the possibility that more than one channel may lead to a $\mathrm{SN}$ Ia, perhaps explaining part of the observed diversity. Interestingly, in the context of supersoft sources, the time that elapses between the merger of two $\mathrm{CO}$ white dwarfs and the actual supernova is $\sim 10^{5} \mathrm{yr}$, and, during this phase, the merged object would look like a supersoft source without a companion star (with $T \simeq 0.5-1 \times 10^{6} \mathrm{~K}$ and $L_{\mathrm{X}} \simeq 10^{37} \mathrm{ergs} \mathrm{s}^{-1}$ ), which could provide a potential test for this channel (Voss \& Nelemans 2008).

\section{Observational Tests of SN Ia Progenitor Models}

\section{The detection of circumstellar material}

Marietta et al. (2000) simulated the interaction of the supernova ejecta with the companion star and showed that a significant fraction of the envelope of the companion ( $\sim 10-20 \%$ for a main-sequence star or subgiant) is stripped from the companion and mixed with the supernova ejecta. Since this material is likely to be dominated by hydrogen (at least in the classical supersoft channel), this should then lead to easily detectable hydrogen emission lines in the nebular phase of the supernova. To date, with the exception of some extremely unusual supernovae (e.g., SN 2002ic; Hamuy et al. 2003), no hydrogen has ever been detected in a normal SN Ia. Indeed, the present lowest upper limits (less than $\sim 0.01-0.02 M_{\odot}$; Leonhard 2007) now provide a strong constraint on the supersoft model, since these limits are not consistent with the Marietta predictions. However, it now seems that the amount of stripping in the original Marietta calculations may have been significantly overestimated and that it is substantially smaller if realistic stellar models for the companion are employed, marginally compatible with the observational limits (Pakmor et al. 2008; also see Meng, Chen \& Han 2007).

More encouragingly, Patat et al. (2007) recently found some direct evidence for circumstellar material in a normal SN Ia, SN 2006X. They observed a variation of $\mathrm{Na}$ lines immediately after the supernova which they interpreted as arising from the ionisation and subsequent recombination of $\mathrm{Na}$ in circumstellar material. This strongly favours a SD progenitor for this supernova, but at present only about $10-$ $20 \%$ of SNe Ia show this behaviour and the interpretation is not unambiguous.

\section{Detecting surviving SN Ia companions}

One of the most direct ways of confirming the SD model would be the discovery of 
the surviving companion that is now a runaway star moving away from the centre of a supernova remnant. Indeed, Ruiz-Lapuente et al. (2004) have claimed to have identified such a companion in the Tycho supernova remnant, a G2IV star with a high peculiar velocity. While such a star is consistent with the SD model, this claim is still quite controversial. Most importantly, the rapid rotation predicted by this model, assuming that the companion is co-rotating with the orbit at the time of the explosion, is not observed (Kerzendorf et al. 2009). This does not yet rule out that this star is the surviving companion, as there is an alternative model in which the star is a stripped, slowly rotating red giant, but this is not apriori very likely.

\subsubsection{Binary Evolution and the Final Fate of Massive Stars}

While it has been clear for many years that binary interactions strongly affect the structures of stellar envelopes, both by mass loss and by mass accretion, and hence are likely to be a major cause for the observed diversity of supernova sub-types, it has only recently become clear that they can also alter the core evolution and, in fact, the final fate of a star. Generically, one expects that, if mass loss/accretion occurs during an early evolutionary phase, the core continues to evolve subsequently like a less or more massive star. However, this is not true if mass loss occurs after the main-sequence phase.

\section{Black Hole or Neutron Star?}

If a star loses its envelope after hydrogen core burning, but before helium ignition (or early during helium core burning; i.e., experiences Case B mass transfer), the evolution of the core can be drastically altered. Because of the lack of a H-burning shell, the convective core does not grow during helium core burning, and stars end up with much smaller CO and ultimately iron cores (Brown et al. 1999, 2001). Indeed, because of this, such H-deficient stars formed in binaries as a result of Case B mass transfer, with initial masses as high as 50/60 $M_{\odot}$ (the exact limit depends on the Wolf-Rayet mass-loss rate), are expected to end their evolution as neutron stars rather than as black holes (Brown et al. 2001), the expected fate for their single-star counterparts. Single stars only become Wolf-Rayet stars if their initial mass is larger than $\sim 25-35 M_{\odot}$ (again dependent on the exact massloss rate). This is larger than the initial mass where single stars are believed to produce black holes $\left(\sim 20-25 M_{\odot}\right.$; e.g., Fryer \& Kalogera 2001). Since the formation of a slowly rotating black hole is not apriori expected to be associated with a bright supernova (as the whole star can just collapse into a black hole), this has the important implication that all normal $\mathrm{H}$-deficient core-collapse supernovae $(\mathrm{SNe} \mathrm{Ib} / \mathrm{Ic})$ may require a close binary companion. 


\section{Electron-Capture Supernovae}

Another mass range where binary interactions can drastically change the final fate of a massive star is near the minimum mass for stars to explode as supernovae (around $7 M_{\odot}$, where the exact value depends on the amount of convective overshooting and the metallicity of the star). Single stars in this mass range experience a second dredge-up phase when they ascend the asymptotic giant branch (AGB), where a large fraction of the H-exhausted core is dredged up and mixed with the envelope. This reduces the core mass at the end of the AGB phase; as a consequence, single stars as massive as $10 / 11 M_{\odot}$ probably produce $\mathrm{ONeMg}$ white dwarfs rather than a supernova. In contrast, if such stars lose their H-rich envelopes due to a binary interaction before reaching the AGB, they end up with much larger He cores and are likely to produce an electron-capture (e-capture) supernova.

An e-capture supernova occurs in a very degenerate $\mathrm{ONeMg}$ core, long before an iron core has developed, and is triggered by the sudden capture of electrons onto Ne nuclei taking away the hydrostatic support provided by the degenerate electrons (Nomoto 1984). This occurs at a characteristic density $\left(\sim 4.5 \times 10^{9} \mathrm{~g} \mathrm{~cm}^{-3}\right.$; Podsiadlowski et al. 2005), which can be related to a critical pre-collapse mass for the $\mathrm{ONeMg}$ core of $\sim 1.37 M_{\odot}$.

\section{A Dichotomous Scenario for Neutron-Star Formation and Neutron-Star Kicks}

The post-collapse (i.e., neutron-star [NS]) mass of an e-capture supernova depends on the equation of state for matter at nuclear density, but has been estimated to be close to $1.25 M_{\odot}$ (Podsiadlowski et al. 2005). This is significantly lower than the NS mass from iron core collapse $\left(\sim 1.35 M_{\odot}\right)$. Indeed, Schwab, Podsiadlowski \& Rappaport (2010) analysed the NS mass distribution of pulsars with very well determined masses and found a bimodal NS mass distribution with a sharp peak at the e-capture mass of $1.25 M_{\odot}$ and a broader distribution around $1.35 M_{\odot}$, lending further support for two NS formation channels.

This also has important implications for neutron-star birth kicks. It has long been known that young pulsars have rather high space velocities. The interpretation of these high velocities is that they must have received a large kick when they were born in the supernova because of an asymmetry in the explosion mechanism. In the most recent study of pulsar birth kicks, Hobbs et al. (2005) found that the natal kick distribution is well approximated by a Gaussian kick distribution with a velocity dispersion of $265 \mathrm{~km} \mathrm{~s}^{-1}$, with no evidence for a low-velocity tail in the distribution. On the other hand, there has been mounting evidence that not all neutron stars receive large kicks at birth (e.g., the eccentricity distribution of Be X-ray binaries [Pfahl et al. 2002b]; the problem of pulsar retention in globular clusters 
[Pfahl, Rappaport \& Podsiadlowski 2002a and references therein] and the properties of the double pulsar, J0737-3039 [Podsiadlowski et al. 2005]). It is tempting to associate the two NS formation channels with different pulsar kick distributions (Podsiadlowski et al. 2004a; also see van den Heuvel 2004). Since, in an e-capture supernova, the whole core collapses, it is relatively easy to eject the rest of the loosely bound envelope. This probably leads to a "fast" supernova explosion, where the instabilities in the accretion shock that are presently the best candidates for the origin of supernova kicks (Blondin \& Mezzacappa 2006, 2007; Foglizzo et al. 2007) did not have time to develop. Indeed, since e-capture supernovae are expected to occur mainly in binary systems (since, as discussed above, a binary may be required to prevent the second dredge-up; Podsiadlowski et al. 2004a), this would also naturally explain why there is no evidence for low-kick neutron stars in the single pulsar population.

\subsubsection{Hypernovae and Gamma-Ray Bursts}

In the late 90s it was realised that, in addition to the normal core-collapse and thermonuclear explosions, there are more energetic supernovae with an energy output $\gtrsim 10^{52}$ ergs, i.e., they are at least 10 times as energetic as a normal supernova. These are now often referred to as hypernovae with the proto-type being SN 1998bw (Iwamoto et al. 1998). Interestingly, at least some of the hypernovae are associated with long-duration gamma-ray bursts (LGRBs), the most powerful explosive events known in the Universe. On the other hand, the nature of their progenitors is almost completely unknown. It is clear that LGRBs are relatively rare events (see Podsiadlowski et al. 2004b and references therein). After correcting for beaming, the LGRB rate in a typical galaxy like ours is $\sim 10^{-5} \mathrm{yr}^{-1}$, where the estimate has an uncertainty of about an order of magnitude. This rate is in fact comparable to the hypernova rate (Podsiadlowski et al. 2004b). This rate implies that less than about 1 in 1000 core-collapse supernovae produces a LGRB and that the production of a LGRB requires some special circumstances; i.e., the progenitors cannot just be more massive single star, but stars that are unusual in some respects, e.g., because of a combination of low-metallicity and rapid rotation (Yoon \& Langer 2005; Woosley \& Heger 2006), or because of binary evolution effects, as I will discuss now.

In the presently favoured collapsar model (Woosley 1993; MacFadyen \& Woosley 1999), a LGRB is triggered by the collapse of a rapidly rotating massive core. In order for the collapse to proceed via a disc phase, the specific angular momentum in the core has to be larger than a few $10^{16} \mathrm{~cm}^{2} \mathrm{~s}^{-1}$. In the case of a single star, this requires that the star for some reason has not been spun down during its evolution, 
the normal fate for most massive star. Alternatively, in a binary scenario it may have been spun up by various binary processes.

\section{Tidal Spin-Up Models}

In many respects the simplest binary process that can produce a rapidly rotating helium star is tidal spin up since, in a tidally locked binary, a star can be spun up (or down) until its spin angular velocity is equal to the orbital angular velocity (e.g., Izzard et al. 2004). Simple angular-momentum estimates suggest that this requires an orbital period shorter than $\sim 10 \mathrm{hr}$ (Podsiadlowski et al. 2004b). In practise, this means that the companion is most likely a compact object (a neutron star or a black hole). Such systems are indeed observed; for example, the X-ray binary Cygnus X-3 contains a Wolf-Rayet star in orbit with a neutron star or black hole (van Kerkwijk et al. 1992). In the case of Cygnus X-3, it is not clear whether the Wolf-Rayet star will ultimately collapse to form a black hole and produce a LGRB. Nevertheless, similar systems that produce a black hole are likely to exist, indeed with a rate compatible with the LGRB rate.

Detmers et al. (2006) have recently modelled the evolution of such systems and, in particular, the spin-up evolution of the companion star. Indeed, they found that tidal spin-up of the core is possible. However, they also showed that, at solar metallicity, the expected strong wind from the Wolf-Rayet star leads to a significant widening of the binary and the ultimate spin-down of the companion. As a consequence, this channel is only likely to work at low metallicity when the wind mass-loss rate is expected to be much lower.

In cases where the Wolf-Rayet companion filled its Roche lobe, Detmers et al. (2006) found that it was then likely that the Wolf-Rayet star would merge completely with the compact companion, quite similar to another LGRB model, proposed originally by Fryer \& Woosley (1999).

\section{Merger Models}

Most binary models for LGRBs proposed to date involve the merger of two stars. This is a particularly efficient way for converting orbital angular momentum into spin angular momentum. A variety of different types of binary mergers can be distinguished depending on the nature of the components and the cause of the merging.

The most widely discussed merger models consider the merging of two compact cores inside a common envelope (e.g., Fryer \& Woosley 1998; Fryer \& Heger 2006), where one of the cores can already be a compact star (e.g., a neutron star or a black hole). One of the most interesting cases involves the merger of the non- 


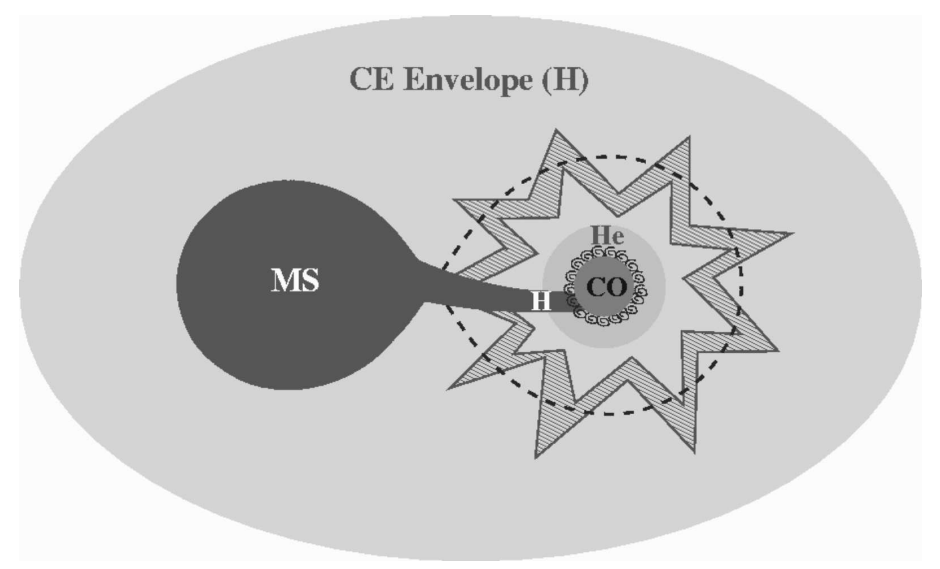

Figure 1.5 Schematic illustration of the process of explosive common-envelope ejection. The H-rich stream from the Roche-lobe-filling immersed companion penetrates deep into the core of the primary, mixing hydrogen into the heliumburning shell. This leads to a thermonuclear runaway ejecting the helium shell and the hydrogen-rich envelope. (From Podsiadlowski et al. 2010.)

degenerate cores of two massive stars. This occurs when the initial masses of the binary components are very close (typically within $\sim 5-10 \%$ ) and both stars already have a compact core at the time of the binary interaction, leading to a doublecore common-envelope phase (as first discussed by Brown 1995; also see Dewi et al. 2006). Statistically, it is more likely that the initially more massive star has already developed a $\mathrm{CO}$ core while the less massive star has a less evolved $\mathrm{He}$ core. When the two cores merge, this will lead to a rapidly rotating object consisting of a $\mathrm{CO}$ core with a helium envelope. Since the merging process is driven by friction within the common hydrogen-rich envelope, it is not entirely clear how the merger can proceed to its conclusion and still eject the hydrogen-rich envelope completely at the same time.

\section{Explosive Common-Envelope Ejection}

A rather different route to a LGRB was discovered by N. Ivanova (Ivanova 2002; Podsiadlowski et al. 2010) when studying the slow merger of two massive stars after helium core burning (i.e., involving Case $\mathrm{C}$ mass transfer). This evolution occurs when mass transfer from a red supergiant to a less massive companion is unstable. This leads to a common-envelope phase where the secondary spirals-in inside the envelope of the original supergiant. At some stage during this spiral-in, the immersed companion itself will fill its Roche lobe and start to transfer mass to the core of the supergiant (as illustrated in Fig. 1.5). Most importantly the stream 
emanating from the secondary, which initially is mainly composed of hydrogenrich material, can penetrate deep into the helium core of the supergiant, eroding it in the process (Ivanova \& Podsiadlowski 2003).

If the initial mass ratio of the binary is relatively large, it can happen that, at some point during the merging process, the H-rich material from the secondary is mixed into the hot helium-burning shell (with a temperature of a few $10^{8} \mathrm{~K}$ ). This leads to a nuclear runaway and the rapid expansion and ultimately the ejection of the $\mathrm{He}$ rich shell and with it of the total H-rich envelope. This mechanism of 'explosive' merging provides a new mechanism for ejecting a common envelope. Unlike the standard case (Paczyński 1976), where the ejection energy is orbital energy, the energy source here is nuclear energy. In order to eject the envelope in a typical case, only a few \% of a solar mass of H-rich material has to be burned, less than found in the actual calculations. Explosive common-envelope ejection provides a new mechanism for $\mathrm{CE}$ ejection that can operate even when the orbital energy is insufficient to eject the envelope otherwise. The stream penetrates particularly deep into the core when the entropy of the secondary is low. This favours relatively low-mass companions with masses less than $\sim 3 M_{\odot}$.

In the context of LGRBs, one implication is that this process predicts that both the hydrogen envelope and the helium-rich layer are ejected and that the final product is a pure $\mathrm{CO}$ core, consistent with the constraint that all LGRB-related supernovae to date are of Type Ic. Furthermore, the $\mathrm{CO}$ core is moderately spun up in the phase where the stream interacts with the helium core (i.e., before the explosive phase). In our calculations, the final specific angular momentum of the core was $\sim 10^{16} \mathrm{~cm}^{2} \mathrm{~s}^{-1}$, consistent with the angular-momentum requirement in the collapsar model. Simple estimates for the rate of this channel suggest a rate of $\sim 10^{-6} \mathrm{yr}^{-1}$, which would be somewhat too low to explain the total local LGRB rate; but this rate is expected to be higher at lower metallicity, where there is a larger orbital-period range for Case $\mathrm{C}$ mass transfer, and it could be much higher if Case D mass, as discussed in $\S 1.3 .4$, also contributes to this channel.

\subsection{Low- and Intermediate-Mass X-Ray Binaries and the Formation of Millisecond Pulsars}

Generically, X-ray binaries are binary systems where a star transfer matter either by Roche-lobe overflow or a stellar wind to a compact companion star, which typically is a neutron star or a black hole. Traditionally, X-ray binaries are divided into two classes, low-mass X-ray binaries (LMXBs; with donor masses $\lesssim 1.5 M_{\odot}$ ), the topic of this section and high-mass X-ray binaries (HMXBs; with donor masses $\gtrsim$ $10 M_{\odot}$ ), the topic of the next section. As I will discuss later, many of the so-called 
LMXBs most likely descended from systems where the donor star had an initial mass of $1.5-4.5 M_{\odot}$, i.e., they really involve intermediate-mass X-ray binaries (IMXBs); I will therefore refer to these in this section collectively as L/IMXBs.

The detailed observational properties and their accretion processes of L/IMXBs and HMXBs are discussed in detail in other chapters of these proceedings. Here I would just like to comment on a common mis-conception considering the different Galactic space distributions of L/IMXBs and HMXBs. Superficially, they appear to be very different: HMXBs are found very close to the Galactic disc, while L/IMXBs have a much broader Galactic latitude distribution; they are therefore often referred to as "bulge sources", and it has even been argued that the majority may have been ejected from globular clusters. However, this is almost certainly not the case. The main difference between these two classes is the difference in kick velocity these systems receive when the neutron star is born and the subsequent lifetime of the system. As discussed in $\S 1.4$, most neutron stars are believed to receive a large kick when they are born in a supernova. In fact, in many cases, the binary system may become unbound as a consequence. In cases where the system remains bound, a binary with a low-mass companion will receive a larger systemic kick than a binary with a high-mass companion (assuming the same NS kick momentum). In addition, because of the longer lifetime, the X-ray binary with the low-mass companion will live longer and hence be able to travel further away from the Galactic plane than a system with a high-mass companion and a much shorter lifetime. Brandt \& Podsiadlowski (1995) modelled the Galactic space distribution of LMXBs and HMXBs and showed that, assuming they are all born in the Galactic plane and adopting the same NS kick distribution for both cases, they could perfectly reproduce the observed space distributions of both classes without the need for invoking different initial populations.

\subsubsection{L/IMXBs Formation Scenarios}

L/IMXBs are typically rather bright X-ray sources (with $L_{\mathrm{X}}>10^{36} \mathrm{ergs} \mathrm{s}^{-1}$ ) and can be seen throughout the Galaxy. There are only about 200 bright L/IMXBs; this already indicates that they must be rather rare objects. Indeed, the formation of an L/IMXB requires a couple of rather improbable steps. Since the typical orbital separation in an L/IMXBs is $0.1-10 R_{\odot}$, much smaller than the size of the NS progenitor, this implies that their formation requires a $\mathrm{CE}$ and spiral-in phase. However, in the case of an LMXB, this implies that the orbital energy released in the spiral-in of the low-mass companion inside the envelope of the NS progenitor has to be enough to eject a rather massive envelope. This is energetically challenging and implies that, in most cases, such binaries are likely to merge completely. Only, if the NS progenitor is a very extended red supergiant at the beginning of 
mass transfer (with a low envelope binding energy) can a low-mass star eject the supergiant's envelope. Even if the system has passed this first hurdle, it still has to survive the supernova in which the neutron star is born. Since generally more than half the total mass of the system is ejected in the supernova, most systems with lowmass companions are likely to be disrupted in the supernova. On the other hand, systems that receive a supernova kick in the right direction (against their orbital motion) have a higher probability of remaining bound (e.g., Brandt \& Podsiadlowski 1995). These constraints are less severe for intermediate-mass companions, strongly favouring them in BPS studies (Pfahl, Rappaport \& Podsiadlowski 2003).

\section{L/IMXBs in globular clusters}

It has long been known that the number of L/IMXBs per unit mass is much larger in globular clusters (GCs) than in the Galaxy, implying a formation rate (per unit stellar mass) that is a factor of $\sim 20$ larger in the GC population and suggesting different formation channels. Indeed, since the stellar densities in GCs are so high, this suggests dynamical formation channels as the explanation for the higher rate.

The dynamical formation process that has been studied the longest involves the tidal capture of a star by a passing neutron star (Fabian, Pringle \& Rees 1975). If a neutron star comes close to a normal star, it induces tidal oscillations in its envelope. The energy of these oscillations is taken from the relative orbital energy of the two stars. Therefore, if the encounter is close enough (the neutron star typically has to come within $\sim 3$ stellar radii), enough energy can be taken out of the orbit to change the relative orbit from an unbound orbit to a bound orbit, i.e., produce a bound NS binary.

The encounter of a neutron star with a pre-existing binary in a globular cluster could be an even more efficient process for producing a NS binary (see, e.g., Davies 1995). This leads to a complicated 3-body interaction, the most likely outcome of which is the ejection of the lightest component of the unstable triple, typically one of the stars in the initial binary, leaving the NS with a new companion. Even though primordial binary systems in globular clusters may be relatively rare, the cross section for a 3-body interaction is the orbital separation of the initial binary, which can be much larger than the cross section required for a tidal capture. Note, however, that this process is likely to produce relatively wide NS binaries. Whether tidal capture or 3-body (and possibly even 4-body) interactions are the dominant formation for L/IMXBs in globular clusters is still the source of much current debate.

\subsubsection{The Origin of Millisecond Pulsars}

Among the population of radio pulsars, there are $\sim 200$ radio pulsars with very short spin periods (as short as $1.4 \mathrm{~ms}$ ) and relatively weak magnetic fields ( $\lesssim$ 
$\left.10^{9} \mathrm{G}\right)$. Unlike the bulk of the normal radio pulsar population, the majority of these are found in binary systems. It is generally believed that these are recycled pulsars which achieved their short spin periods by accretion of mass and angular momentum from a companion star (see, e.g., Bhattacharya \& van den Heuvel 1991).

However, it has also now been established for more than two decades (Ruderman et al. 1989) that the standard model, where their evolution is considered similar to the evolution of cataclysmic variables (CVs; systems similar to LMXBs but where the compact object is a white dwarf), cannot explain some of the main observed characteristics of L/IMXBs.

\section{Problems with the Standard Model}

Two of the main problems with the standard model for LMXBs (e.g., Ruderman et al. 1989; Bhattacharya \& van den Heuvel 1991) are that it cannot explain their distributions of orbital periods (which is qualitatively different from the CV distribution) and X-ray luminosities. The typical luminosities, and hence mass-transfer rates, appear to be about an order of magnitude larger than the standard model predicts. A further problem with the standard model, referred to as the 'birthrate' problem for ms pulsars, is that the birthrate of LMXBs appears to be a factor of 10 to 100 lower than the birthrate of ms pulsars both in the Galactic disc (Kulkarni \& Narayan 1988; Johnston \& Bailes 1991) and in globular clusters (Fruchter \& Goss 1990; Kulkarni, Narayan \& Romani 1990).

\subsubsection{The Case of Cyg X-2: the Importance of Intermediate-Mass X-ray Binaries}

Until about a decade ago, intermediate-mass X-ray binaries (IMXBs) had received fairly little attention (see, however, Pylyser \& Savonije 1988, 1989). This changed with several new developments. First, Davies \& Hansen (1998) studying dynamical interactions in globular clusters found that IMXBs are much easier to form dynamically than LMXBs and speculated that these IMXBs, which do not exist in globular clusters at the present epoch, might be the progenitors of the observed millisecond (ms) pulsars rather than the presently observed LMXBs.

The second development was a re-assessment of the evolutionary status of the Xray binary Cyg X-2. Spectroscopic observations of Cyg X-2 by Casares, Charles \& Kuulkers (1998) combined with the modeling of the ellipsoidal light curve (Orosz \& Kuulkers 1999) showed that the secondary in Cyg X-2 was a low-mass star of $\sim 0.6 \pm 0.13 M_{\odot}$ that was much hotter and almost a factor of 10 too luminous to be consistent with a low-mass subgiant with an orbital period of 9.84 days (see Podsiadlowski \& Rappaport 2000). The explanation for this paradox was found 

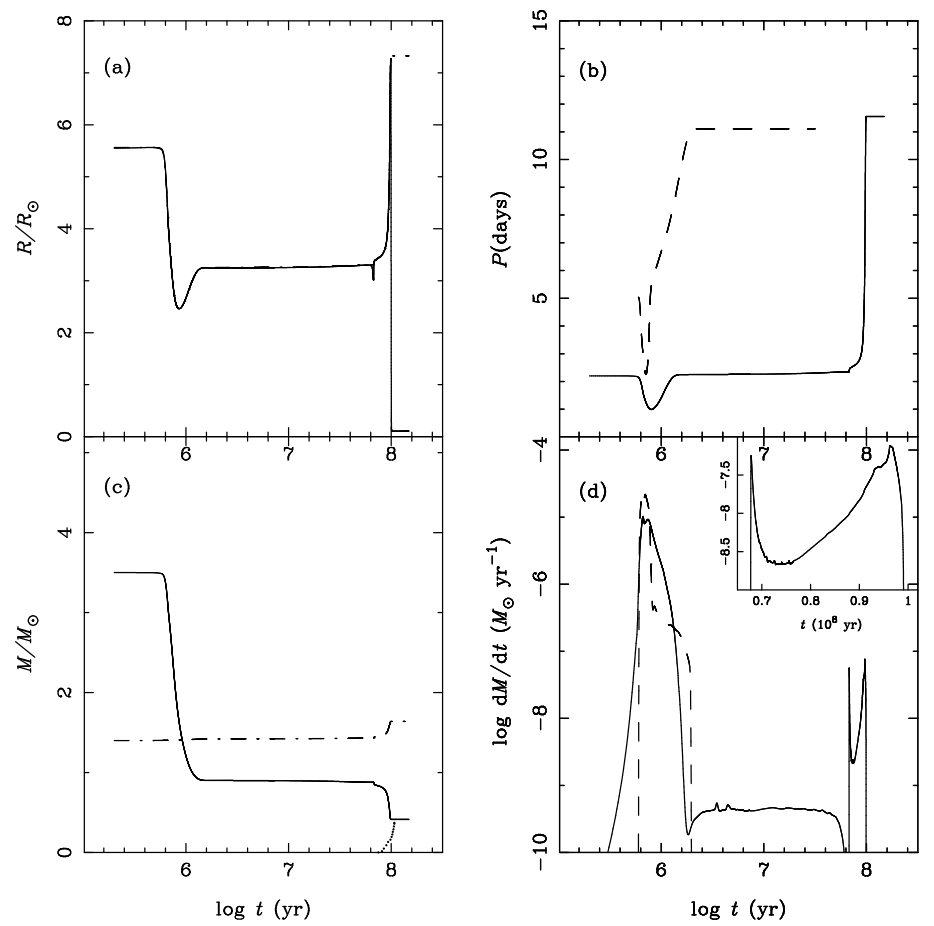

Figure 1.6 Key binary parameters as a function of time (with arbitrary offset) in a binary calculation illustrating the possible evolution of Cygnus X-2. The calculation assumes that mass transfer started when the secondary was near the end of the main sequence (case AB mass transfer). Panel (a): radius (solid curve) and Roche-lobe radius (dot-dashed curve) of the secondary; panel (b): the orbital period (solid curve); panel (c): the mass of the secondary (solid curve) and the primary (dot-dashed curve); panel (d): the mass-loss rate from the secondary (solid curve); the inset shows a blow-up of the second slow mass-transfer phase (hydrogen shell burning). The dashed curves in panel (b) and (d) show the orbital period and mass-transfer rate for a case B calculation. (From Podsiadlowski \& Rappaport 2000.)

independently by King \& Ritter (1999) and Podsiadlowski \& Rappaport (2000) (also see Kolb et al. 2000; Tauris, van den Heuvel \& Savonije 2000) who showed that the characteristics of Cyg X-2 can best be understood if the system was the descendant of an IMXB where the secondary initially had a mass of $\simeq 3.5 M_{\odot}$ and lost most of its mass in very non-conservative case AB or case B mass transfer (see Fig. 1.6). Thus, Cyg X-2 provides observational proof that IMXBs can eject most of the mass that is being transferred from the secondary (perhaps in the form of an equatorial outflow as is observed in SS 433 [Blundell et al. 2001]) and subsequently resemble classical LMXBs. This immediately suggests that a large fraction of socalled 'LMXBs' may in reality be IMXBs or their descendants. 


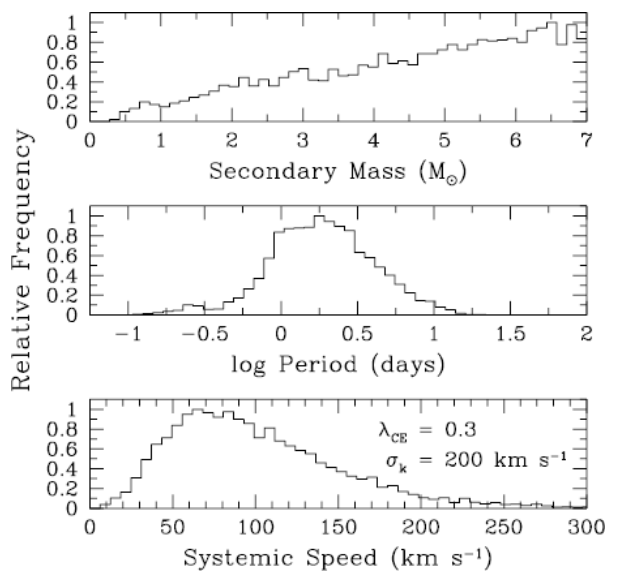

Figure 1.7 The initial properties of L/IMXBs at the beginning of the masstransfer phase. From top to bottom: initial secondary mass distribution, orbital period distribution and system velocity. (From Pfahl et al. 2003.)

No. 2, 2003

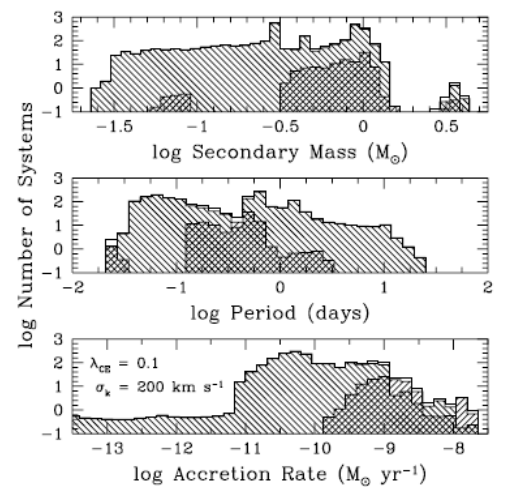

1043

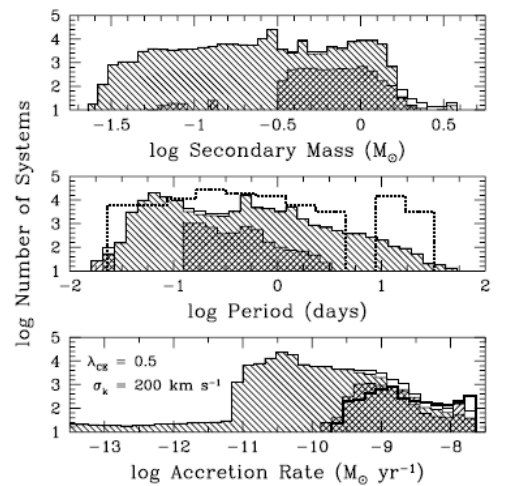

Figure 1.8 The present properties of L/IMXBs. From top to bottom: secondary (donor) mass distribution, orbital-period distribution and NS mass-accretion rate. The panels on the left assume a lower (but probably more realistic) efficiency for the CE ejection process than the panels on the right (parametrised using a lower envelope structure parameter $\lambda_{\mathrm{CE}}$ ). The double hatched regions indicate persistent X-ray sources, the single hatched regions transients. The thick, solid distribution on the bottom right panel illustrates how the inclusion of X-ray irradiation effects may affect the theoretical $\dot{M}$ distribution. (From Pfahl et al. 2003.)

\subsubsection{Modeling the L/IMXB Population}

In order to assess the importance of IMXBs, Podsiadlowski, Rappaport \& Pfahl (2002) [PRP] and Pfahl et al. (2003) carried out a comprehensive study consisting of two parts. The first involved a series of $\sim 150$ binary evolution calculations 
using a realistic binary evolution code (PRP). These calculations covered the mass range of 0.8 to $7 M_{\odot}$ and all evolutionary phases from early case A to late case $\mathrm{B}$ mass transfer. The second part involved the integration of these binary sequences into a state-of-the-art Monte-Carlo BPS code which allowed a detailed comparison of the calculations with observations (Pfahl et al. 2003).

Fig. 1.7 shows the initial properties of systems that become X-ray binaries. As the top panel shows, the majority of X-ray binaries initially have intermediate-mass companions, simply because these are much easier to form. However, since the initial mass-transfer phase for IMXBs is very rapid (see Fig. 1.6), systems are most likely to be observed after the secondaries have transferred/lost a large fraction of their mass and essentially look like standard 'LMXBs' (although they may show evidence for $\mathrm{CNO}$ processing and He-enrichment in their envelopes). The panels of Figure 1.8 show the distributions of the secondary mass, orbital period and neutronstar mass-accretion rate during the X-ray binary phase at the current epoch. Unlike the initial mass distribution, the mass distribution at the current epoch is dominated by relatively low-mass systems, and there are hardly any systems above $\sim 2 M_{\odot}$ because of the initial high mass-transfer rate for IMXBs. The orbital-period distribution shows no period gap and extends to very short periods. Finally, the luminosity distribution displays a fairly strong peak around $5 \times 10^{-11} M_{\odot} \mathrm{yr}^{-1}$ and has a sharp cut-off at $\sim 10^{-11} M_{\odot} \mathrm{yr}^{-1}$.

While these distributions show many of the characteristics of the observed distributions of 'LMXBs', in fact more so than a model that only includes CV-like systems, there are still some fairly obvious discrepancies. First, there are too many short-period systems to be consistent with the observed period distribution (e.g., Ritter \& Kolb 1998). Second, while the distribution of mass-accretion rate (and hence X-ray luminosity) has a sharp cut-off at $\sim 10^{-11} M_{\odot} \mathrm{yr}^{-1}-$ as is desirable, the peak in the distribution is probably too low by about an order of magnitude.

\section{Irradiation-Driven Mass-Transfer Cycles}

Our binary evolution calculations at the moment do not account for irradiation effects of the secondary that can dramatically change the evolution of the system either by irradiation-driven winds (Ruderman et al. 1989) or irradiation-driven expansion of the secondary (Podsiadlowski 1991). Podsiadlowski (1991) showed that, if a star with a convective envelope is irradiated by a sufficiently high X-ray flux (so that hydrogen is being ionised), it will try to expand by a factor of 2 to 4 . If such a star is already filling its Roche lobe, this will drive mass transfer at a highly enhanced rate on a timescale determined by the thermal timescale of the convective envelope. These early calculations assumed spherical illumination; this is unrealistic in a binary situation since, in this case, the energy that drives the expansion 

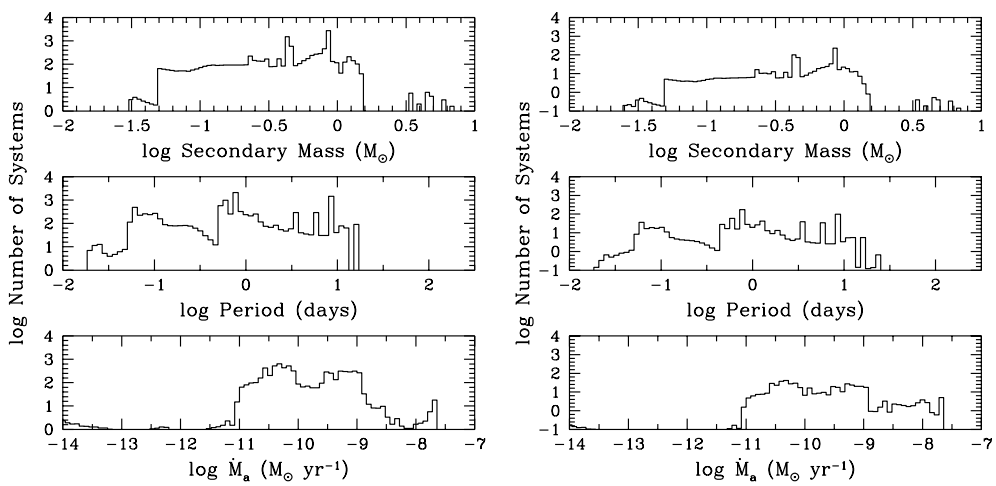

Figure 1.9 The distributions of secondary mass (top panel), orbital period (middle panel), and neutron-star mass-accretion rate at the current epoch for a standard reference model (left panels) and a model simulating irradiation-driven masstransfer cycles. In the latter, it is assumed that during phases of irradiation-driven mass transfer, the mass-transfer is a factor of 10 larger than the secular masstransfer rate with correspondingly shorter lifetimes for the X-ray active phase. The standard model on the left predicts $\sim 2000$ observable X-ray binaries in the Galaxy, while the model with irradiation cycles predicts $\sim 200$ systems, more consistent with the observed sample. (From Podsiadlowski, Rappaport \& Pfahl 2003b.)

can, in principle, be redirected to the unilluminated side where it can be radiated away. But even then one expects a moderate expansion which can lead to masstransfer cycles (Hameury et al. 1993), characterised by relatively short phases of enhanced mass transfer and long detached phases. These would not only increase the mass-transfer rates during the X-ray-active portion of the cycles but also reduce the duration of the X-ray active lifetime of these systems by a proportionate amount. This could provide a simultaneous solution of the X-ray luminosity and the birthrate problem.

To simulate this in our BPS simulations, we have taken the binary sequences calculated with our standard assumptions, but assumed that the mass-transfer rate was a factor 10 larger during X-ray active phases than in the standard calculation and was interrupted by long X-ray quiet phases, which we calculated in such a way that the secular evolution of the systems (e.g., the orbital period, secondary mass as a function of time) were the same as in the standard sequences; this procedure is consistent with the results of Hameury et al. (1993), but in some sense assumes that irradiation effects are relatively modest.

The panels on the right in Fig. 1.9 show the results of such a simulation. As one would expect, there are now many systems with higher mass-transfer rates, 
consistent with observations, and the number of observable systems is reduced by a factor of 10 to $\sim 200$, again in better agreement with the actual numbers.

\subsection{High-Mass X-Ray Binaries}

\subsubsection{Systems with Neutron Stars}

The mass-transfer process in high-mass X-ray binaries (HXMBs) is in some respects quite different from those of L/IMXBs. If the compact object is a neutron star, Roche-lobe overflow will always become unstable because of the large mass ratio. In general, one distinguishes two main modes of mass transfer: atmospheric Roche-lobe overflow and wind mass transfer.

Atmospheric Roche-lobe overflow

Because there is an atmosphere above the photosphere of a star, mass transfer generally starts somewhat before the photosphere reaches the Roche lobe. Since, for low-mass main-sequence stars (but not for giants!), the radial extent of this atmosphere is very small, this initial phase is not usually very important. However, for intermediate-mass stars, and even more so for high-mass stars, the extent can be substantial and a large amount of mass can be transferred in this phase of atmospheric Roche-lobe overflow (see Figure 12 in PRP). In particular, if mass transfer ultimately becomes unstable, this is the only phase in which such a system can be observed as an X-ray source. The mass-transfer rate, in this phase, can be orders of magnitude larger than the Eddington mass-accretion rate. ${ }^{8}$ Therefore these systems typically appear as very bright X-ray sources.

\section{Wind mass transfer}

In addition, massive stars have strong stellar winds and part of this wind can be accreted by the companion. Because of gravitational focusing, the accretion cross section is generally much larger than the geometric cross section of the accretor; it is given by the Bondi-Hoyle accretion radius

$$
R_{\mathrm{BH}}=\frac{2 G M}{v^{2}},
$$

where $M$ is the mass of the accretor and $v$ the relative velocity between the wind and the accreting object (assumed to be supersonic here). For a spherically symmetric wind emanating from the donor with mass-loss rate $\dot{M}_{\text {wind }}$, it is easy to

\footnotetext{
8 The Eddington mass-accretion rate for an accreting object is the rate at which it has to accrete so that the accretion luminosity equals the Eddington limit at which radiation pressure stops accretion:

$\dot{M}_{\mathrm{Edd}}=4 \pi c R / \kappa$, where $R$ is the radius of the accreting object and $\kappa$ is the opacity of the material being accreted. For a neutron star of $1.4 M_{\odot}$, this accretion rate is $\simeq 2 \times 10^{-8} M_{\odot} \mathrm{yr}^{-1}$.
} 
show that the fraction of the wind accreted by the companion is given by

$$
\frac{\dot{M}_{\text {acc }}}{\dot{M}_{\text {wind }}}=\left(\frac{v_{\text {orb }}}{v_{\text {wind }}}\right)^{4}\left(\frac{M_{\text {acc }}}{M_{\text {acc }}+M_{\text {donor }}}\right)^{2},
$$

where $v_{\text {orb }}$ is the orbital velocity and the subscripts 'acc' and 'donor' refer to the donor and accretor. For an HMXBs, the last factor in this expression is generally a small number and hence only a small fraction of the wind can be accreted in most cases. As a consequence, wind-accreting HMXBs tend to be fainter then those that accrete by atmospheric Roche-lobe overflow.

\section{Be X-ray binaries}

A third class of HMXBs are Be X-ray binaries, where the mass donor is a $\mathrm{Be}$ star. Be stars are rapidly rotating stars, rotating at a speed close to break-up. Be $\mathrm{X}$-ray binaries are believed to be systems where the first mass-transfer phase (in the phase before the formation of the neutron star) was stable and the companion star was able to accrete a substantial amount of mass, being spun up to break-up in the process. Because of their large rotation rates, Be stars tend to have substantial winds and eject matter episodically. This ejection is strongly concentrated towards their equatorial planes. Once a neutron star has formed in a supernova, the neutron star can accrete part of this wind and appear as an X-ray source. In particular, if the orbit of the neutron star is eccentric (e.g., due to a supernova kick), these X-ray outbursts tend to be very transient. Be X-ray binaries probably form the largest sub-group of HMXBs but, because of their transient nature, their total number is somewhat uncertain.

\section{The Final Fate of HMXBs Containing Neutron Stars}

As already mentioned, mass transfer in an HMXB is generally expected to become unstable because of the large mass ratio. The reason is not only that the massratio is above some critical value, but also because there is an additional instability, the Darwin instability. The neutron star orbiting the massive star induces a tide in the massive star which will try to spin it up, so that ultimately it would spin in co-rotation with the neutron star's orbit. However, for a sufficiently large mass ratio, there is not enough angular momentum in the neutron star's orbit to bring the massive star into co-rotation..$^{9}$ But the transfer of angular momentum from the orbit to the star makes the orbit shrink and forces the neutron star to merge with the massive star and form a common envelope.

\footnotetext{
9 The exact criterion for the Darwin instability is that a binary system is unstable if the moment of inertia of the star being spun up (assuming solid-body rotation) is larger then $1 / 3$ of the momentum inertia of the orbit, i.e., $I_{\text {star }}>1 / 3 \mu A^{2}$, where $\mu$ is the reduced mass of the binary and $A$ the orbital separation.
} 


\section{The formation of Thorne-Żytkow Objects}

The fate of his CE phase depends on whether the orbital energy released in the spiral-in is enough to eject the envelope or not. If the initial orbital period is relatively short ( $\lesssim 1 \mathrm{yr}$; Terman, Taam \& Hernquist 1995), such systems are expected to merge completely; this means that the neutron star sinks to the centre, replacing or disrupting whatever there was before. Such objects with neutron cores are known as Thorne-Żytkow objects (TŻOs; Thorne \& Żytkow 1975, 1977). These objects will appear as very cool red supergiants. Since these are difficult to distinguish from normal red supergiants, it is presently not clear whether they actually exist. Since this is the possible fate for the majority of known HMXBs, their birthrate in the Galaxy is expected to be quite high $\left(\sim 2 \times 10^{-4} \mathrm{yr}^{-1}\right.$; Podsiadlowski, Cannon $\&$ Rees 1995). Depending on the uncertain lifetime of this phase (limited, e.g., by the wind mass-loss rate), a few to $10 \%$ of all red supergiants with a luminosity comparable to or above the Eddington limit for a neutron star could harbour neutron cores. One way of distinguishing them from normal red supergiants is through their anomalously large abundances of proton-rich elements, in particular molybdenum (Mo; Biehle 1991; Cannon 1993).

Once criticism that has been raised against the very existence of TŻOs (see, e.g., Chevalier 1993) is that, during the initial spiral-in phase, the accretion rate onto the neutron star may occur in the neutrino-dominated regime where all the accretion energy is radiated away in the form of neutrinos and becomes hypercritical (i.e., can exceed the photon Eddington limit by an arbitrary amount). If this were the case, one would expect the neutron star to accrete enough matter to be converted into a black hole. The resulting object would presumably be a black hole surrounded by a massive disc. ${ }^{10}$

\section{The formation of double neutron star binaries}

If the orbital period of the HMXB is relatively long ( $~ 1 \mathrm{yr}$ ), the orbital energy released by the spiralling-in neutron star is expected to be sufficient to eject the common envelope. The post-CE system will be a much closer binary consisting of the neutron star (assuming that it did not experience supercritical accretion and was converted into a black hole) in orbit with the hydrogen-exhausted core of the massive star, i.e., a helium star (see the left panel of Fig. 1.10). If the helium star is sufficiently massive $\left(\gtrsim 4 M_{\odot}\right)$, it will appear as a Wolf-Rayet star with a very powerful, optically thick wind (with $\dot{M}_{\text {wind }} \gtrsim 10^{-6} M_{\odot} \mathrm{yr}^{-1}$ ). If even a small fraction of this wind is accreted by the neutron star, the system will again appear

10 If this disc is sufficiently massive it is conceivable that self-gravitating objects, even low-mass stars, could form in such a disc due to gravitational instabilities (Podsiadlowski et al. 1995). 

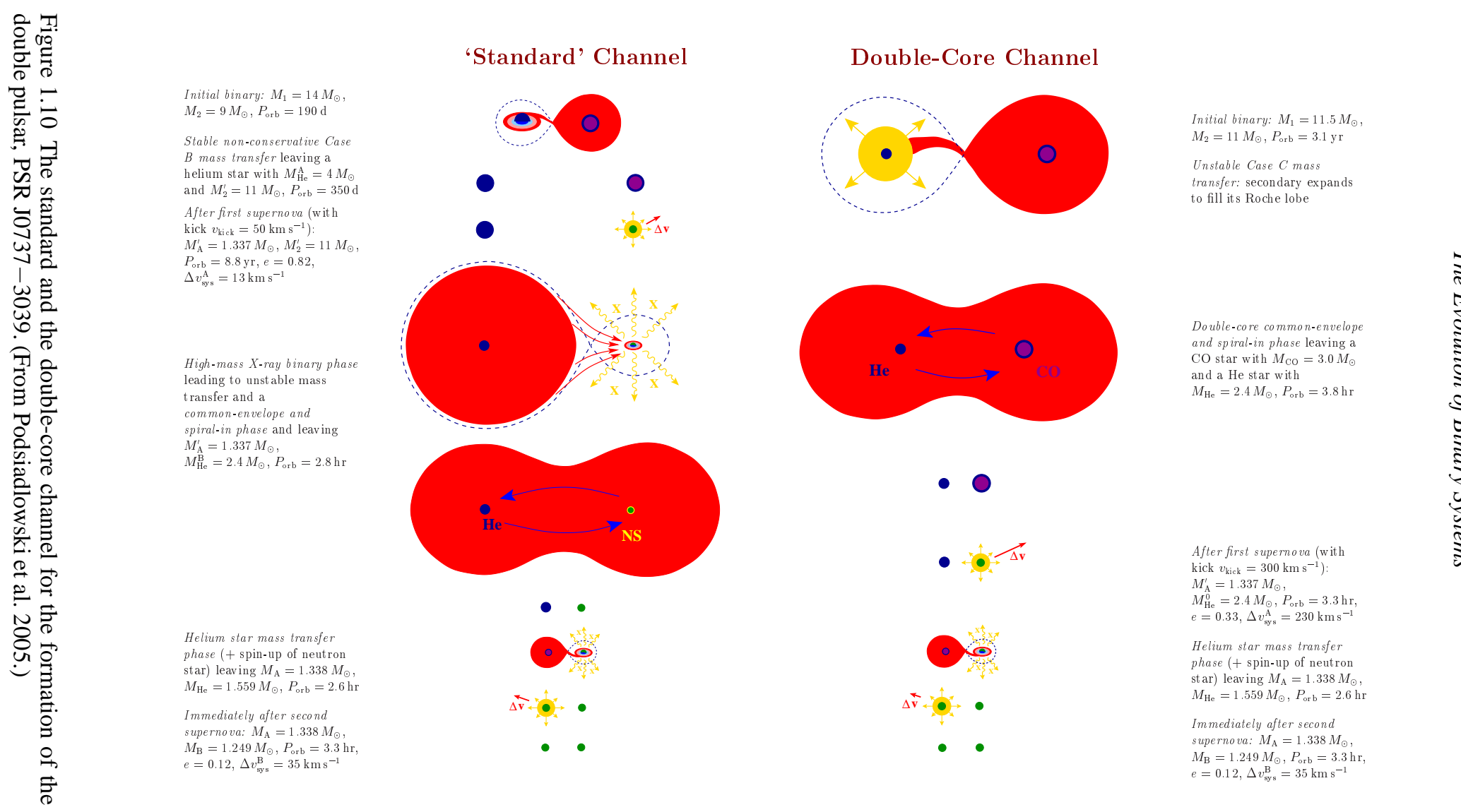

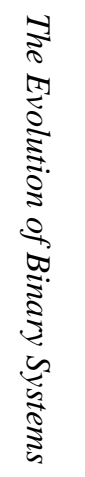


as a bright X-ray source. Cyg X-3 with an orbital period of $4.8 \mathrm{hr}$ provides a prototypical example for this type of system. ${ }^{11}$

Eventually, the helium star will explode in a supernova (of Type Ib/Ic) and itself produce a neutron star. Because of the natal NS supernova kick, there is a high probability that the system becomes disrupted in this second supernova. If this is the case, both neutron stars (one a young pulsar, the other a relatively old neutron star) will move apart as runaway neutron stars with velocities comparable to their final orbital velocities in the disrupted binary (typically a few $100 \mathrm{~km} \mathrm{~s}^{-1}$ ). On the other hand, if the system remains bound, the surviving system is a binary containing two neutron stars. The first double neutron-star (DNS) system discovered was the Hulse-Taylor pulsar, PSR 1913+16 (Hulse \& Taylor 1975), with an orbital period of about $8 \mathrm{hr}$ and a spin period of $59 \mathrm{~ms}$ (the pulsar was mildly spun up [recycled] by accretion from the helium star in the previous He-star binary phase). Since the original discovery, half a dozen more DNS systems have been found (see, e.g., the list in Schwab et al. 2010). These systems have become extremely important probes of fundamental physics. The orbital evolution of these systems is entirely driven by gravitational radiation. Since the neutron stars are too small to interact in any way (even tidally), the resulting measured decrease of the orbital period in these systems provides a direct and very accurate test of Einstein's theory of General Relativity (for this achievement, Hulse and Taylor were awarded the Nobel prize in 1993).

In addition, if the orbital period of a DNS system is short enough $(\lesssim 10 \mathrm{hr})$, gravitational radiation will bring the system together in a Hubble time making the two neutron stars merge in a final cataclysmic event. Such merger events are accompanied by a major burst of gravitational waves, which could be directly detectable with current and future gravitational wave experiments (e.g., Advanced LIGO). If such a merger occurs in a relatively nearby galaxy, such mergers should be detectable within the next few years.

In addition, such mergers are likely to produce a burst of gamma rays, and mergers of two neutron stars or a neutron star and a black hole are presently the best candidates for short-duration GRBs. ${ }^{12}$ Finally, DNS mergers may also be the source of some unusual nucleosynthesis: in particular, because of the overabundance of neutrons, they are a potential source for all the neutron-rich, r-process elements in the Universe, for which no source has yet been identified unambiguously. ${ }^{13}$

As mentioned before, it is not clear whether a neutron star spiralling-in inside a

11 Note, however, that, in the case of Cyg X-3, it is not clear whether the compact object is a neutron star or a black hole.

12 Unlike long-duration GRBs, the average duration of a short-duration GRB is less than $1 \mathrm{~s}$.

13 Explosive nucleosynthesis in supernovae is often considered a possible source for r-process elements, but it is still unclear whether the conditions for the r-process are right for a sufficiently long time during the explosive supernova phase. 
massive envelope will survive as a neutron star or be converted into a black hole by hypercritical accretion. If this were the case, the above scenario could not produce a DNS system. An alternative scenario to produce DNSs was proposed by Brown (1995), which is shown in the right panel of Figure 1.10. If the masses of the initial binary are sufficiently close (typically within $4 \%$ ) and mass transfer occurs when the primary has already finished helium core burning (Case $\mathrm{C}$ mass transfer), the secondary will already have finished its hydrogen-core-burning phase and developed a helium core. In this case, one may expect a common-envelope phase, where the common envelope contains the envelopes of both stars, and the embedded binary consists of the He-exhausted core of the primary and the $\mathrm{H}$-exhausted core of the secondary (so-called double-core evolution). Once the common envelope is ejected, the system has become a close binary with two $\mathrm{H}$-exhausted stars, one most likely containing a $\mathrm{CO}$ core, the other a He core. After two supernovae, the system will end up as a DNS system. Even though this evolution requires rather special circumstances, Dewi, Podsiadlowski \& Sena (2006) found that, within the substantial uncertainties of this channel, this channel could account for a large fraction of DNS systems and potentially all.

The Double Pulsar: PSR J0737-3039

A particularly important recent discovery is the binary pulsar PSR J0737-3039 (Burgay et al. 2003), which consists of two pulsars: one old, recycled pulsar with a spin period of $22.7 \mathrm{~ms}$ and one younger pulsar with a spin period of $2.77 \mathrm{~s}$ (Fig. 1.10 illustrates two possible evolutionary histories for the double pulsar). Because of the short orbital period of this system $(2.4 \mathrm{hr})$, general relativistic effects are much more important than in the Hulse-Taylor pulsar, and this system has now become our most important laboratory for testing general relativity (see Kramer \& Stairs 2008). Note also that the second-born pulsar (Pulsar B) has a mass of $1.249 M_{\odot}$, very close to the mass expected for an e-capture supernova. Indeed, there is strong evidence that the second-born neutron star did not receive a large kick: (a) the orbit is almost circular, (b) the system space velocity is small and (c) the spin of the recycled pulsar (Pulsar A) is aligned with the orbit (see Podsiadlowski et al. 2005 for further discussion).

\subsubsection{X-ray Binaries Containing Black Holes}

Interlude: do black holes exist?

These days we often take the existence of black holes for granted, but we should ask whether it has actually been proven. In the case of stellar-mass black holes, the argument is usually just based on the mass of the compact object, since the 
maximum mass of a neutron star is believed to be less than $\sim 3 M_{\odot}$. The inferred masses for the compact objects in some of the "black-hole" binaries, as largely determined by their mass function (eq. 1.1), exceeds $10 M_{\odot}$ in some of the best cases (e.g., GRS 1915+105; Greiner, Cuby \& McCaughrean 2001). While this is well in excess of the maximum neutron-star mass, it does not prove that the compact object is a black hole as the equation of state of matter at these densities is very poorly understood, and it is has been postulated that other states of matter could exist (e.g., involving strange matter, Q-balls, etc.) that do not have a maximum mass limit.

What one needs to show the existence of a black hole is the existence of an event horizon, the defining feature of a black hole. This is possible in principle, if one has a system where one knows the accretion rate onto a compact object, but one does not see the accretion luminosity that would be associated with this accretion rate, as all the mass-energy disappears below the event horizon without trace (in contrast, for objects with a surface, most of that energy has to be radiated away). This is possible in principle, but has not yet been demonstrated convincingly (at least to this author), despite some claims in the literature.

\section{The Origin of Black-Hole Binaries with Low-Mass Companions}

A large fraction of the black-hole $(\mathrm{BH})$ binaries known to date appear to contain low-mass donor stars, in many cases resembling LMXBs except that they often tend to be transient rather than persistent X-ray sources (see, e.g., Lee, Brown \& Wijers 2002). This poses an immediate problem concerning their formation. As already discussed in the context of the formation of LMXBs, it is challenging for a low-mass star to eject the massive envelope of a NS progenitor in the $\mathrm{CE}$ phase. This becomes even harder for a $\mathrm{BH}$ progenitor that is necessarily more massive. Indeed, if one uses realistic envelope structures, the maximum orbital energy available from the spiral-in of a low-mass star falls short by about a factor of $5-10$ to what is needed to eject the envelope of a BH progenitor (see Podsiadlowski, Rappaport \& Han 2003 for details). This problem has long been known (even though it is often ignored!), and a number of solutions have been proposed. These include: (1) the models of the envelopes of massive red supergiants may be wrong (in particular, due to uncertainties in the wind mass loss); (2) the modeling of the CE phase may be in error (this would not be surprising considering that this is a very poorly understood phase; however, energy conservation should not be violated); (3) there are alternative exotic formation scenarios, involving triple systems (Eggleton \& Verbunt 1986) or the formation of a low-mass star in the debris disc of, e.g., a Thorne-Żytkow object (Podsiadlowski et al. 1995: Podsiadlowski et al. 2003a); (4) the companions of these systems may have descended from intermediate-mass objects (similar to the case of most LMXBs; Podsiadlowski et 

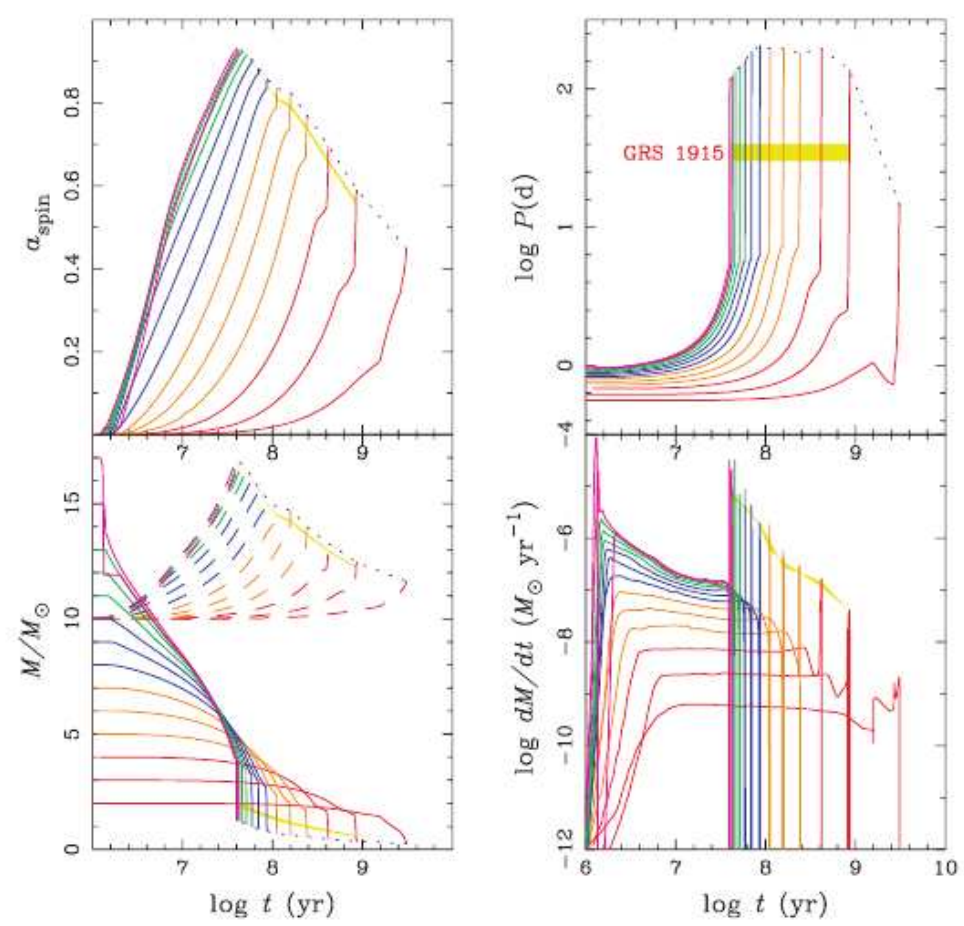

Figure 1.11 Selected properties of black-hole binary sequences as a function of time since the beginning of mass transfer: black-hole spin parameter (top left), orbital period (top right), black-hole mass and secondary mass (dashed and solid curves, bottom left), mass-transfer rate (bottom right). In all sequences, the black hole has an initial mass of $10 M_{\odot}$ and is initially non-rotating. The secondaries (mass donors) range from 2 to $17 M_{\odot}$ and are initially unevolved (the larger the initial mass of the secondary, the shorter the duration of the mass-transfer phase). The shaded regions in each panel indicate the period range of 30 to $40 \mathrm{~d}$ (similar to the orbital period of GRS $1915+105$ with $\mathrm{P}_{\text {orb }}=33.5 \mathrm{~d}$ ). (From Podsiadlowski et al. 2003a.)

al. 2003a; Justham, Rappaport \& Podsiadlowski 2006); or (5) the energy source for the ejection of the common envelope is nuclear energy rather than orbital energy ("explosive common-envelope ejection"; Podsiadlowski et al. 2010 and $\S 1.4$ ). At present, there is no consensus on the resolution of this conundrum.

\section{The Evolution of Black-Hole Binaries}

In X-ray binaries where the accreting compact source is a black hole, the masstransfer process and the overall evolution differs significantly in some respects from the case of neutron-star binaries. 
If the donor star is a low-mass star, the main processes are similar to the case of LMXBs, except that, because of the larger mass of the black hole, the masstransfer rate tends to be lower. One consequence of this is that most black-hole binaries with low-mass companions are X-ray transients with alternating phases of high and low accretion rates depending on the state of the accretion disc (see the corresponding chapters in these proceedings).

For black-hole binaries with massive companions, the main difference to NS systems is that standard Roche-lobe overflow is not necessarily unstable for mass ratios as high as $\sim 2$. Therefore, these systems may experience long phases of stable mass transfer as bright $\mathrm{X}$-ray sources.

Podsiadlowski et al. (2003a) have systematically explored the evolution of blackhole binaries. Figure 1.11 shows some of the key results from this study. It shows the evolution of initially unevolved main-sequence stars (ranging from 2 to $17 M_{\odot}$ initially), transferring mass to a black hole with an initial mass of $10 M_{\odot}$. In all of these sequences, mass transfer is stable at all times (even a model with $20 M_{\odot}$ was only marginally unstable). In systems where the initial donor mass exceeds the black-hole mass, mass transfer initially occurs on a thermal timescale, leading to very high mass-transfer rates and the spikes in the $\dot{M}$ distribution in Figure 1.11. After the mass ratio has been reversed, mass transfer continues to be driven by the nuclear evolution of the donor star. Since this phase is much longer-lived than the thermal timescale phase, BH X-ray binaries are most likely to be observed in this phase. As the donor ascends the giant branch, the mass-transfer rate goes up again, producing another spike in the $\dot{M}$ evolution.

The main results of this study were: (1) RLOF in BH X-ray binaries is stable for mass ratios as high a 2, and, because of the mass loss, the X-ray active phase can be much longer than the lifetime of a single star of the same initial mass. (2) Even if mass accretion onto the black-hole is Eddington limited, the black holes can accrete substantial amounts of mass (see the bottom left panel of Fig. 1.11); hence the present observed BH mass is not necessarily a good indicator of the initial post-collapse BH mass. (3) Black holes can also accrete substantial amounts of angular momentum (top left panel of Fig. 1.11) and be spun up in the process to spin parameters $a \sim 0.4-0.9$ (assuming that the black holes were initially non-rotating).

\section{The Nature of Ultraluminous X-ray Sources}

Ultraluminous X-ray sources (ULXs; Fabbiano 1989) are typically defined as Xray sources that have a luminosity exceeding $L_{\mathrm{X}}=10^{39} \mathrm{ergs} \mathrm{s}^{-1}$, which is roughly the Eddington luminosity for a $10 M_{\odot}$ black hole (the exact definition varies somewhat from author to author). The particular interest of these systems is that they 

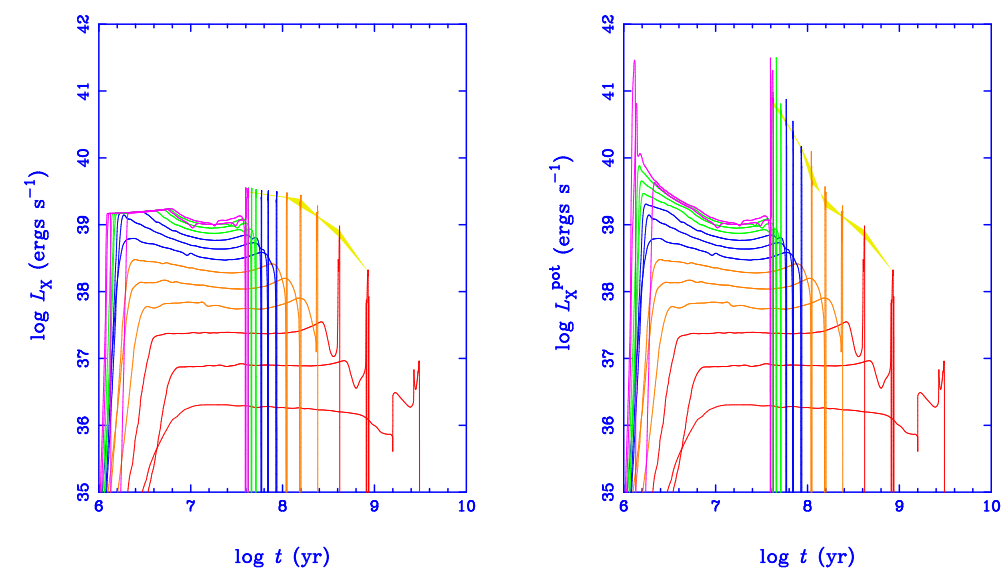

Figure 1.12 X-ray luminosities, assuming Eddington-limited accretion, (left panel) and potential $\mathrm{X}$-ray luminosities, assuming non-Eddington-limited accretion, (right panel) for the binary evolution sequences in Figure 1.11 as a function of time since the beginning of mass transfer. (From Podsiadlowski et al. 2003a.)

may harbour intermediate-mass black holes with masses of $10^{2}-10^{3} M_{\odot}(\mathrm{Col}-$ bert \& Mushotzky 1999) which could possibly represent the missing link between stellar-mass black holes and supermassive black holes at the centres of galaxies. Indeed, they could be the key building block for supermassive black holes. As discussed in detail in the chapter by Fabbiano in these proceedings, it is now clear that probably the vast majority of ULXs are physically associated with regions of very active massive star formation (such as in the Antennae interacting galaxies and the Cartwheel galaxy). This strongly suggests that most of them (but not necessarily all!) are linked to massive BH binary populations. Indeed, there is no problem to feed black holes at a mass-transfer rate at which they could appear as ULXs. This is shown in Figure 1.12 in which the right panel shows the potential X-ray luminosity of the evolutionary sequences in Figure 1.11 (for comparison, the left panel shows the luminosity in these sequences if accretion were Eddington limited). The potential X-ray luminosity is defined as the accretion luminosity one would observe if all the mass lost from the donor star were accreted by the black hole. Note that the potential luminosities can reach values as high as $\sim 10^{41} \mathrm{ergs} \mathrm{s}^{-1}$, albeit for only very short periods of time.

In this context, I would like to clear up a common mis-conception in the literature. One often hears that stellar BH X-ray binaries may look like ULXs if they are in a thermal timescale mass-transfer phase. However, as Figure 1.12 shows, these systems reach potential ULX luminosities for the more massive companions during most of the phases where mass transfer is driven by the nuclear evolution of the 
donor. ${ }^{14}$ Since this phase is many orders of magnitude longer-lived, it is much more likely that the observed ULXs are in this phase rather than the thermal timescale phase (the very short initial spikes in Fig. 1.12). (For recent detailed studies of massive BH binaries and their relation to ULXs see Rappaport, Podsiadlowski \& Pfahl 2005; Madhusudhan et al. 2006, 2008.)

The main issue with ULXs being stellar-mass BH binaries is that they have to accrete at a rate that exceeds the Eddington accretion rate by a significant factor (for some of the more luminous systems by a factor up to $\sim 20$ ). It has been suggested that, in magnetic accretion discs, such high accretion rates can be reached, but the exact physical mechanism remains unclear and may involve a photon bubble instability (Begelman 2002, 2006; Ruszkowski \& Begelman 2003), emission from a hot, optically thin corona magnetically coupled to the accretion flow (Socrates \& Davis 2006) or something else. Until we understand the physics of magnetic accretion discs better, this issue remains unresolved (also see Hawley's chapter in these proceedings).

14 In addition, beaming, both geometric (King et al. 2001) and relativistic (Körding, Falcke \& Markoff 2002), may increase the apparent luminosities of these systems further if they are observed along the direction where the radiation is beamed. 


\section{References}

[1] Begelman, M. 2002, Astrophys. J., 568, 97.

[2] Begelman, M. 2006, Astrophys. J., 643, 1065.

[3] Bhattacharya, D., and van den Heuvel, E. P. J. 1991, Phys. Rep., 203, 1.

[4] Biehle, G. T. 1991, Astrophys. J., 380, 167.

[5] Blondin, J. M., and Mezzacappa, A. 2006, Astrophys. J., 642, 401.

[6] Blondin, J. M., and Mezzacappa, A. 2007, Nature, 445, 58.

[7] Blundell, K. M., Mioduszewski, A. J., Muxlow, T. W., Podsiadlowski, Ph., and Rupen, M. P. 2001, Astrophys. J., 562, L79.

[8] Brandt, W. N. and Podsiadlowski, Ph. 1995, Month. Not. R. Astron. Soc., 274, 461.

[9] Brown, G. E. 1995, Astrophys. J., 440, 270.

[10] Brown, G. E., Heger, A., Langer, N., Lee, C.-H., Wellstein, S., and Bethe, H. A. 2001, New Astron., 6, 457.

[11] Brown, G. E., Lee, C.-H., and Bethe, H. A. 1999, New Astron., 4, 313.

[12] Burgay, M., et al. 2003, Nature, 426, 531.

[13] Burrows, C., et al. 1995, Astrophys. J., 452, 680.

[14] Cannon, R. C. 1993, Month. Not. R. Astron. Soc., 263, 817.

[15] Cappellaro, E., Turatto, M. 1997, in Thermonuclear Supernovae, ed. P. RuizLapuente et al. (Kluwer, Dordrecht), p. 77.

[16] Casares, J., Charles, P., and Kuulkers, E. 1998, Astrophys. J., 493, L39.

[17] Chevalier, R. A. 1993, Astrophys. J., 411, L33.

[18] Colbert, E., and Mushotzky, R. 1999, Astrophys. J., 519, 89.

[19] Davies, M. B. 1995, Month. Not. R. Astron. Soc., 276, 887.

[20] Davies, M. B., and Hansen, B. M. S. 1998, Month. Not. R. Astron. Soc., 301, 15.

[21] Detmers, R. G., Langer, N., Podsiadlowski, Ph., and Izzard, R. G. 2008, Astron. \& Astrophys., 484, 83.

[22] Dewi, J. D. W., Podsiadlowski, Ph., and Sena, A. 2006, Month. Not. R. Astron. Soc., 368, 1742.

[23] Dewi, J. D. M., and Tauris, T. M. 2000, Astron. \& Astrophys., 360, 1043.

[24] Duquennoy, A., and Mayor, M. 1991, Astron. \& Astrophys., 248, 485.

[25] Eggleton, P. P. 1983, Astrophys. J., 268, 368.

[26] Eggleton, P. P., and Verbunt, F. 1986, Month. Not. R. Astron. Soc., 220, 13.

[27] Fabbiano, G. 1989, Ann. Rev. Astron. Astrophys., 27, 87.

[28] Fabian, A. C., Pringle, J. E., and Rees, M. J. 1975, Month. Not. R. Astron. Soc., 172, 15 P.

[29] Faulkner, J. 1971, Astrophys. J., 170, L99. 
[30] Fedorova, A. V., Tutukov, A. V., and Yungelson, L. R. 2004, Astronomy Letters, 30, 73.

[31] Foglizzo, T., Galletti, P., Scheck, L., and Janka, H.-T. 2007, Astrophys. J., 654, 1006.

[32] Frankowski, A, and Jorissen, A. 2007, Baltic Astronomy, 16, 104.

[33] Fruchter, A. S., and Goss, W. M. 1990, Astrophys. J., 365, L63.

[34] Fryer, C. L., and Heger, A. 2006, Astrophys. J., 623, 302

[35] Fryer, C. L., and Kalogera, V. 2001, Astrophys. J., 554, 548.

[36] Fryer, C. L., and Woosley, S. E. 1998, Astrophys. J., 502, L9.

[37] Greiner, J., Cuby, J. G., and McCaughrean, M. J. 2001, Nature, 414, 522.

[38] Hachisu, I., Kato, M., Nomoto, K., and Umeda, H. 1999, Astrophys. J., 519, 314.

[39] Hameury, J. M., King, A. R., Lasota, J. P., and Raison, F. 1993, Astron. \& Astrophys., 277, 81.

[40] Hamuy, M., et al. 2003, Nature, 424, 651.

[41] Han, Z., and Podsiadlowski, Ph., 2004, Month. Not. R. Astron. Soc., 350, 1301.

[42] Han, Z., Podsiadlowski, Ph., and Eggleton, P. P. 1995, Month. Not. R. Astron. Soc., 272, 800 .

[43] Han, Z., Podsiadlowski, Ph., and Lynas-Gray, A. E. 2007, Month. Not. R. Astron. Soc., 380, 1098.

[44] Han, Z., Podsiadlowski, Ph., Maxted, P. F. L., Marsh, T. R., and Ivanova, N. 2002, Month. Not. R. Astron. Soc., 336, 449.

[45] Han, Z., Podsiadlowski, Ph., Maxted, P. F. L., \& Marsh, T. R. 2003, Month. Not. R. Astron. Soc., 341, 669.

[46] Hjellming, M. S., and Webbink, R. F. 1987, Astrophys. J., 318, 794.

[47] Hobbs, G., Lorimer, D. R., Lyne, A. G., and Kramer, M. 2005, Month. Not. R. Astron. Soc., 360, 974.

[48] Howell, D. A., et al. 2006, Nature, 443, 308.

[49] Hulse, R. A., and Taylor, J. H. 1975, Astrophys. J., 195, L51.

[50] Iben, I., Jr., and Livio, M. 1993, Publ. Astron. Soc. Pacific, 105, 1373.

[51] Iben, I., Jr., and Tutukov, A. V. 1984, Astrophys. J. Suppl., 54, 335.

[52] Ivanova, N. 2002, D.Phil. Thesis (Oxford University).

[53] Ivanova, N., and Podsiadlowski 2003, in From Twilight to Highlight: the Physics of Supernovae, ed. W. Hillebrandt \& B. Leibundgut (Berlin, Springer), p. 19.

[54] Iwamoto, K., et al. 1998, Nature, 395, 672.

[55] Izzard, R. G., Ramirez-Ruiz, E., and Tout, C. A. 2004, Month. Not. R. Astron. Soc., 348, 1215.

[56] Janka, H.-Th., Langanke, K., Marek, A., Martınez-Pinedo, G., and Mü ller, B. 2007, Phys. Rev., 442, 38.

[57] Johnston, S., and Bailes, M. 1991, Month. Not. R. Astron. Soc., 252, 277.

[58] Justham, S., Rappaport, S., and Podsiadlowski, Ph. 2006, Month. Not. R. Astron. Soc., 366, 1415.

[59] Karovska, M., Schlegel, E., Hack, W., Raymond, J. C., and Wood, B. E. 2005, Astrophys. J., 623, L137.

[60] Kerzendorf, W., et al. 2009, Astrophys. J., 701, 1665.

[61] King, A. R., Davies, M. B., Ward, M. J., Fabbiano, G., and Elvis, M. 2001, Astrophys. J., 552, L109.

[62] King, A. R., and Ritter, H. 1999, Month. Not. R. Astron. Soc., 309, 253.

[63] Kobulnicky, H. A., and Fryer, C. L. 2007, Astrophys. J., 670, 747.

[64] Körding, E., Falcke, H., and Markoff, S. 2002, Astron. \& Astrophys., 382, L13

[65] Kolb, U., Davies, M. B., King, A., and Ritter, H. 2000, Month. Not. R. Astron. Soc., 317, 438 . 
[66] Kramer, M., and Stairs, I. H. 2008, Ann. Rev. Astron. Astrophys., 46, 541.

[67] Kulkarni, S. R., and Narayan, R. 1988, Astrophys. J., 335, 755.

[68] Kulkarni, S. R., Narayan, R., and Romani, R. W. 1990, Astrophys. J., 356, 174.

[69] Kulkarni, S. R., et al. 2007, Nature, 447, 458.

[70] Landau L. D., and Lifshitz, E. M. 1959, The Classical Theory of Fields (Oxford, Pergamon Press).

[71] Lee, C.-H., Brown, G. E., and Wijers, R. A. M. J. 2002, Astrophys. J., 575, 996.

[72] Leonhard, D. C. 2007, Astrophys. J., 670, 1275.

[73] MacFadyen, A. I., and Woosley, S. E. 1999, Astrophys. J., 524, 262.

[74] Madhusudhan, N., Justham, S., Nelson, L., Paxton, B., Pfahl, E., Podsiadlowski, Ph., and Rappaport, S. 2006, Astrophys. J., 640, 918.

[75] Madhusudhan, N., Rappaport, S., Podsiadlowski, Ph., and Nelson L. 2008, Astrophys. J., 688, 1235.

[76] Marietta, E., Burrows, A., and Fryxell, B. 2000, Astrophys. J. Suppl., 128, 615.

[77] Meng, X., Chen, X., and Han, Z. 2007, Publ. Astron. Soc. Japan, 59, 835.

[78] Meyer, F., and Meyer-Hofmeister, E. 1979, Astron. \& Astrophys., 78, 167.

[79] Mezzacappa A., Bruenn, S. W., Blondin, J. M., Hix, W. R., and Bronson Messer O. E. 2007 in The Multicolored Landscape of Compact Objects and their Explosive Origins, ASP Conf. Proc., 924, p 234.

[80] Mikołajewska, J. 2007, Baltic Astronomy, 16, 1.

[81] Morris, T., and Podsiadlowski, Ph. 2007, Science, 315, 1103.

[82] Napiwotzki, R., et al. 2002, Astron. \& Astrophys., 386, 957.

[83] Nelemans, G., Yungelson, L. R., Portegies Zwart, S. F., and Verbunt, F. 2001, Astron. \& Astrophys., 365, 491.

[84] Nomoto, K. 1982, Astrophys. J., 253, 798.

[85] Nomoto, K. 1984, Astrophys. J., 277, 791.

[86] Nomoto, K., Iben, I., Jr. 1985, Astrophys. J., 297, 531.

[87] Orosz, J. A., and Kuulkers, E. 1999, Month. Not. R. Astron. Soc., 305, 132.

[88] Paczyński, B. 1976, in Structure and Evolution of Close Binaries, ed. P. P. Eggleton, S. Mitton \& J. Whelan (Dordrecht, Kluwer), p 75.

[89] Pakmor, R., Röpke, F. K., Weiss, A., and Hillebrandt, W. 2008, Astron. \& Astrophys., 489, 943.

[90] Pasquali, A., Nota, A., Langer, N., Schulte-Ladbeck, R. E., and Clampin, M., 2000, Astron. J., 119, 1352.

[91] Patat, F., et al. 2007, Science, 317, 924.

[92] Perlmutter, S., et al. 1999, Astrophys. J., 517, 565.

[93] Pfahl, E., Rappaport, S., and Podsiadlowski Ph. 2002a, Astrophys. J., 573, 283.

[94] Pfahl, E., Rappaport, S., Podsiadlowski, Ph., and Spruit, H. 2002b, Astrophys. J., 574, 364.

[95] Pfahl, E., Rappaport, S., and Podsiadlowski, Ph. 2003, Astrophys. J., 597, 1036.

[96] Phillips, M. M. 1993, Astrophys. J., 413, L105.

[97] Piersanti, L., Gagliardi, S., Iben, I., Jr., and Tornambé, A. 2003, Astrophys. J., 598, 1229.

[98] Podsiadlowski, Ph. 1991, Nature, 350, 136.

[99] Podsiadlowski Ph., 2001, in Evolution of Binary and Multiple Star Systems, ed. Ph. Podsiadlowski et al. (San Francisco, PASP), ASP Conf. Proc. 229, 239.

[100] Podsiadlowski, Ph., Cannon, R. C., and Rees, M. J. 1995, Month. Not. R. Astron. Soc., 274, 485.

[101] Podsiadlowski, Ph., Dewi, J. D. M., Lesaffre, P., Miller, J. C., Newton, W., and Stone, J. R. 2005, Month. Not. R. Astron. Soc., 361, 1243. 
[102] Podsiadlowski, Ph., Ivanova, N., Justham, S., and Rappaport, S. 2010, Month. Not. R. Astron. Soc., 406, 840

[103] Podsiadlowski, Ph., and Joss, P. C. 1989. Nature, 338, 401.

[104] Podsiadlowski, Ph., Joss, P. C., and Hsu, J. J. L. 1992, Astrophys. J., 391, 246.

[105] Podsiadlowski, Ph., Langer, N., Poelarends, A. J. T., Rappaport, S., Heger, A., and Pfahl, E. 2004a, Astrophys. J., 612, 1044.

[106] Podsiadlowski, Ph., Mazzali, P. A., Nomoto, K., Lazzati, D., and Cappellaro, E. 2004b, Astrophys. J., 607, L17.

[107] Podsiadlowski, Ph., and Mohamed, S. 2007, Baltic Astronomy, 16, 26.

[108] Podsiadlowski, Ph., Morris, T., and Ivanova N. 2006, in Stars with the B[e] phenomenon, ed. M. Kraus, \& A. S. Miroshnichenko (ASP, San Francisco), ASP Conf. Ser., 355, p. 259.

[109] Podsiadlowski, Ph., Morris, T. S., and Ivanova, N. 2007, in SN 1987A: 20 Years After, AIP Conf.Proc., 937, p. 125.

[110] Podsiadlowski, Ph., and Rappaport, S. 2000, Astrophys. J., 529, 946.

[111] Podsiadlowski, Ph., Rappaport, S., and Han, Z. 2003a, Month. Not. R. Astron. Soc., 341, 385.

[112] Podsiadlowski, Ph., Rappaport, S., and Pfahl, E. D. 2002, Astrophys. J., 565, 1107 [PRP].

[113] Podsiadlowski, Ph., Rappaport, S., and Pfahl, E. 2003b, in Radio Pulsars, ed. M. Bailes, D. J. Nice \& S. E. Thorsett, ASP Conf. Proc., 302, p. 283.

[114] Pylyser, E. H. P., and Savonije, G. J. 1988, Astron. \& Astrophys., 191, 57.

[115] Pylyser, E. H. P., and Savonije, G. J. 1989, Astron. \& Astrophys., 208, 52.

[116] Rappaport, S., Podsiadlowski, Ph., and Pfahl, E. 2005, Month. Not. R. Astron. Soc., 356, 401.

[117] Riess, A. G., et al. 1998, Astron. J., 116, 1009.

[118] Ritter, H., and Kolb, U. 1998, Astron. \& Astrophys. Suppl., 129, 83.

[119] Ruszkowski, M., and Begelman, M. C. 2003, Astrophys. J., 586, 384.

[120] Ruderman, M., Shaham, J., and Tavani, M. 1989, Astrophys. J., 336, 507.

[121] Ruiz-Lapuente, P., et al. 2004, Nature, 431, 1069.

[122] Schwab, J., Podsiadlowski, Ph., and Rappaport, S., 2010, Astrophys. J., 719, 722.

[123] Smith, N., Davidson, K., Gull, T. R., Ishibashi, K., and Hellier, D. J. 2003, Astrophys. J., 586, 432.

[124] Socrates, A., and Davis, S. W. 2006, Astrophys. J., 651, 1049.

[125] Spruit, H. C., and Taam, R. E. 2001, Astrophys. J., 548, 900.

[126] Taam, R. E., and Sandquist, E. L. 2000, Ann. Rev. Astron. Astrophys., 38, 113.

[127] Tauris, T. M., van den Heuvel, E. P. J., and Savonije, G. J. 2000, Astrophys. J., 530, L93.

[128] Terman, J. L., Taam, R. E., and Hernquist, L. 1995, Astrophys. J., 445, 367.

[129] Thorne K. S., and Żytkow, A. N. 1975, Astrophys. J., 199, L19.

[130] Thorne K. S., and Żytkow, A. N. 1977, Astrophys. J., 212, 832.

[131] Tylenda, R., and Soker, N. 2006, Astron. \& Astrophys., 451, 223.

[132] van den Heuvel, E. P. J. 2004, in ESA SP 552, 5th INTEGRAL Workshop on the INTEGRAL Universe, ed. V. Schoenfelder, G. Lichti and C. Winkler (Nordwijk; ESA), p. 185.

[133] van den Heuvel, E. P. J., Bhattacharya, D., Nomoto, K., and Rappaport, S. 1992, Astron. \& Astrophys., 262, 97.

[134] van Kerkwijk, M. H., et al. 1992, Nature, 355, 703.

[135] van Rensbergen, W., De Loore, C., and Jansen, K. 2006, Astron. \& Astrophys., 446, 1071. 
[136] Verbunt, F., and Zwaan, C. 1981, Astron. \& Astrophys., 100, L7.

[137] Voss, R., and Nelemans, G. 2008, Nature, 451, 802.

[138] Wampler, E. J., et al. 1990, Astrophys. J., 362, L13.

[139] Webbink, R. F. 1984, Astrophys. J., 277, 355.

[140] Webbink, R. F. 1986, in Critical Observations versus Physical Models for Close Binary Systems, ed. K.-C. Leung \& D. S. Zhai (New York, Gordon \& Breach), p 403.

[141] Whelan, J., and Iben, I., Jr. 1973, Astrophys. J., 186, 1007.

[142] Woosley, S. E. 1993, Astrophys. J., 405, 273.

[143] Woosley, S. E., and Heger, A. 2006, Astrophys. J., 637, 914.

[144] Yoon, S.-C., and Cantiello, M. 2010, Astrophys. J., 717, L62.

[145] Yoon, S.-C., and Langer, N. 2004, Astron. \& Astrophys., 419, 623.

[146] Yoon, S.-C., and Langer, N. 2005, Astron. \& Astrophys., 443, 643.

[147] Yoon, S.-C., Podsiadlowski, Ph., and Rosswog, S. 2007, Month. Not. R. Astron. Soc., 390, 933.

[148] Yungelson, L. R., Livio, M., Tutukov, A. V., and Saffer, R. 1994, Astrophys. J., 420, 336.

[149] Zickgraf, F.-J., et al. 1996, Astron. \& Astrophys., 309, 505. 